\title{
WestVirginiaUniversity
}

THE RESEARCH REPOSITORY @ WVU

Graduate Theses, Dissertations, and Problem Reports

2001

\section{Sensor technology for the Breast Examination Training Instrument}

Bratislav Matic

West Virginia University

Follow this and additional works at: https://researchrepository.wvu.edu/etd

\section{Recommended Citation}

Matic, Bratislav, "Sensor technology for the Breast Examination Training Instrument" (2001). Graduate Theses, Dissertations, and Problem Reports. 1295.

https://researchrepository.wvu.edu/etd/1295

This Thesis is protected by copyright and/or related rights. It has been brought to you by the The Research Repository @ WVU with permission from the rights-holder(s). You are free to use this Thesis in any way that is permitted by the copyright and related rights legislation that applies to your use. For other uses you must obtain permission from the rights-holder(s) directly, unless additional rights are indicated by a Creative Commons license in the record and/ or on the work itself. This Thesis has been accepted for inclusion in WVU Graduate Theses, Dissertations, and Problem Reports collection by an authorized administrator of The Research Repository @ WVU. For more information, please contact researchrepository@mail.wvu.edu. 
Sensor Technology for the Breast Examination Training Instrument

Bratislav Matic

Thesis submitted to the

College of Engineering and Mineral Resources

At West Virginia University

in partial fulfillment of the requirements

for the degree of

Master of Science

in

Electrical Engineering

Committee

Roy Nutter, Ph.D., Chair

Hany Ammar, Ph.D.

Bojan Cukic, Ph.D.

Susan Leight, Ed.D., Guest

Department of Computer Science and Electrical Engineering

Morgantown, West Virginia

2001

Copyright 2001 Bratislav Matic 


\begin{abstract}
Sensor Technology for the Breast Examination Training Instrument (BETI)

Bratislav Matic

Latest medical research has shown high percentage of American women, diagnosed with breast cancer. If detected in its early stage, cancer can be treated successfully. Breast examination might be obtained through three procedures: breast selfexamination (BSE), clinical breast examination (CBE) and mammography. Project BETI was developed with idea of educating and training women and health care professionals in BSE. This instrument consists of a silicone breast model, tactile sensors and serial communication capability. Software was developed for a generic PC that supports an integrated system including:

an interactive video demonstrating BSE techniques, a practical examination using the instrumented breast model, a non-subjective evaluation of a practice BSE examination, and a summary of the user's results from the examination and the user's risk category concerning breast cancer.

This device is designed to be fully automated and to be easily operated by anyone who is literate and can use a computer mouse.
\end{abstract}




\section{TABLE OF CONTENTS}

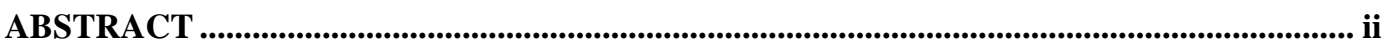

TABLE OF CONTENTS ............................................................................................................ ii

ACKNOWLEDGEMENT ....................................................................................................................v

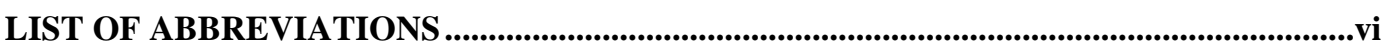

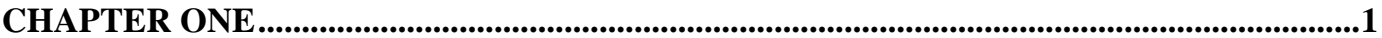

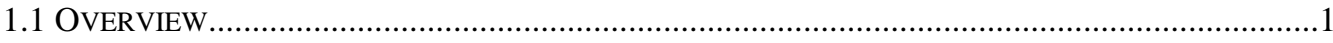

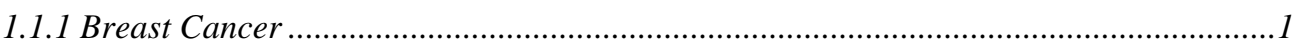

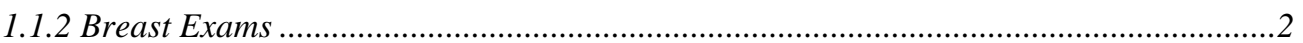

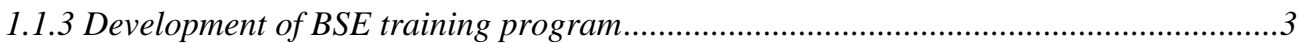

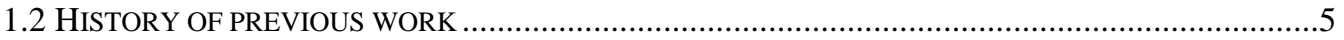

1.3 WORK PROPOSAL

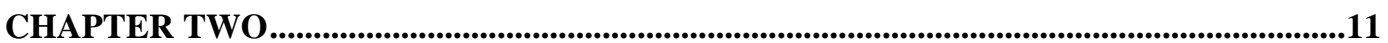

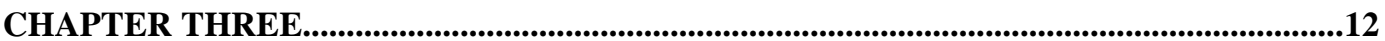

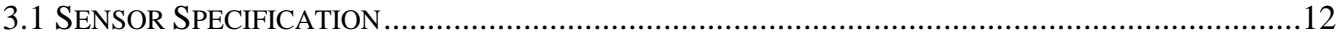

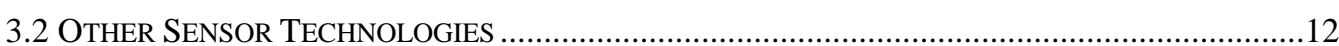

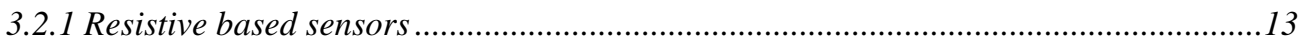

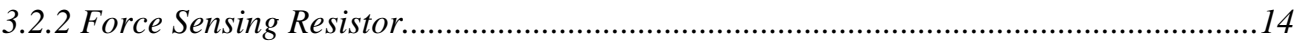

3.2.3 Piezoelectric Polymers...........................................................................................14

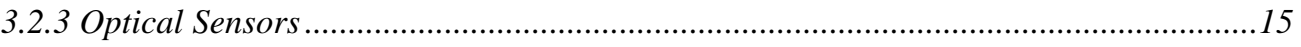

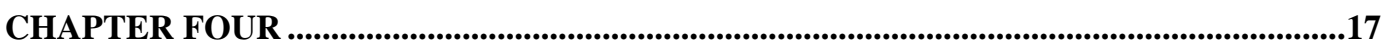

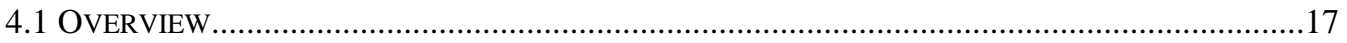

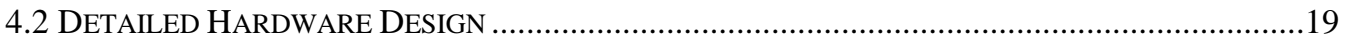

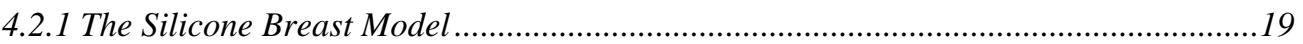

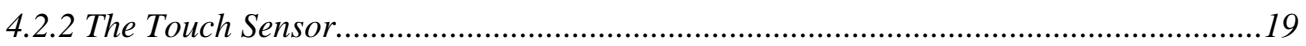

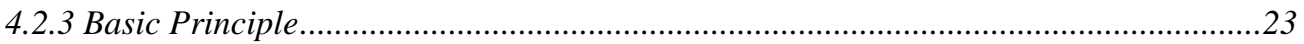

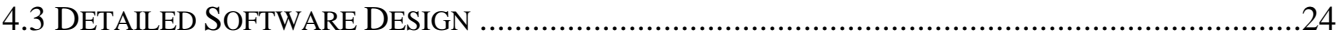

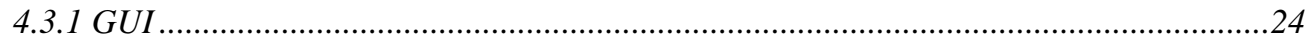

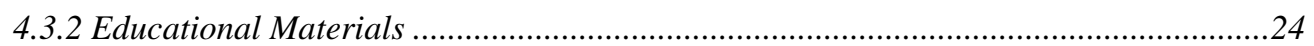




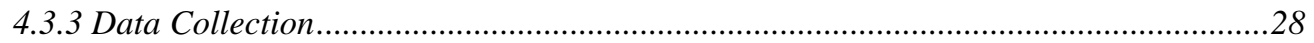

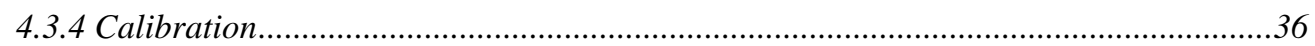

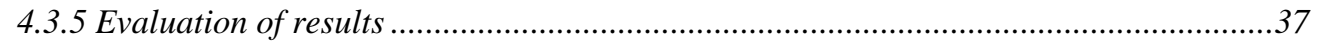

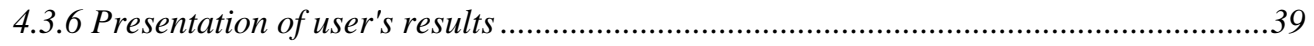

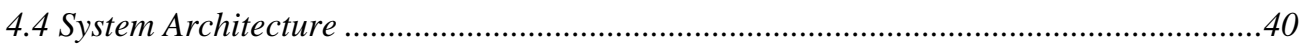

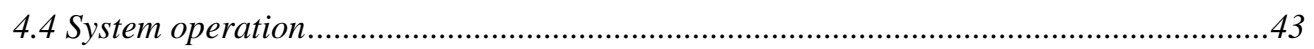

CHAPTER FIVE

CHAPTER SIX ....................................................................................................................................48

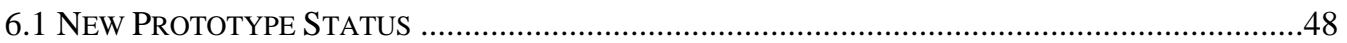

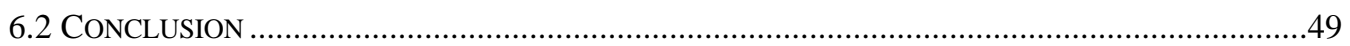

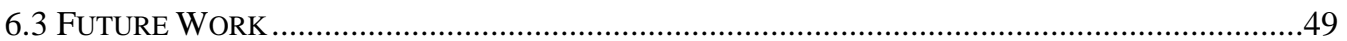




\section{ACKNOWLEDGEMENT}

There are many people that the author wishes to acknowledge for helping to make this thesis a reality. First, I would like to thank Dr. Roy Nutter and Dr. Susan Leight for giving me the opportunity to work on this project, as well as for their support and help in writing this Thesis. In addition, I would like to thank Dr. Hany Ammar and Dr. Bojan Cukic who have encouraged my research work during my graduate studies at West Virginia University.

A continued support from my wife Dragana, is the most valuable gift that any person can get in his/her lifetime. Thousands of miles away from us, our families were all the time with us in our hearts; giving us love and spiritual support.

I also whish to thank my friends Dejan Desovski and Weena Gaulin for all suggestions and help they gave me while working on my Thesis.

Finally, the last but not the least thanks to our precious little cat Cici, who was always with me when I needed support. 


\section{LIST OF ABBREVIATIONS}

$\begin{array}{ll}\text { BETI } & \text { Breast Examination Training Instrument } \\ \text { BSE } & \text { Breast Self-Examination } \\ \text { CBE } & \text { Clinical Breast Examination } \\ \text { GUI } & \text { Graphic User Interface } \\ \text { PC } & \text { Personal Computer } \\ \text { LVDT } & \text { Linear Voltage Displacement Transducer } \\ \text { SBM } & \text { Silicone Breast Model } \\ \text { API } & \text { Application Programming Interface }\end{array}$




\section{CHAPTER ONE}

\section{INTRODUCTION}

\subsection{Overview}

This project develops new hardware and software for training people to do breast exams. This work is an extension of the Breast Examination Training Instrument, originally published by Dr. Sue Leight [1]. This work simplifies and improves the reliability of device as well as provides a marketable product.

The BETI project is based on the development of an instrumented breast model and fully automated training procedure for breast examination. The objective of this application is twofold: to educate users on breast cancer and breast care and to make them capable of implementing techniques of breast examination.

\subsubsection{Breast Cancer}

One woman out of a group of eight, has a high chance of getting breast cancer during her life [2]. In addition, statistical results have shown more than 178,000 new cases of breast cancer diagnosed among American women in 1998. More than 43,000 women die from this disease each year [2]. In the year of 2000, there were 182,800 new cases of breast cancer [16]. So far, no medication or successful procedure has been discovered that would cure $100 \%$ of the discovered breast cancers. If breast cancer is detected and treated in its early stage, the percentage of survivors increases. The main reason for developing this application was to improve early detection of breast cancer.

Breast cancer can be detected by observing of the changes within the breast. The evolution of breast cancer has been classified in three stages: localized, regional and distant.

In the localized stage, lumps are small (diameter is less than $2 \mathrm{~cm}$ ) and the breast cancer is not spread beyond the breast. If detected in this stage, breast cancer can be treated successfully resulting in a survival rate of $97 \%$ [2]. 
During the next stage of cancer development, breast lumps become bigger in their size (usually 2 to $5 \mathrm{~cm}$ ) and the cancer tends to spread to the lymph nodes in the armpit area. Survival rate drops to $77 \%$ [2] if cancer is detected and treated during this stage.

Finally, the distant stage of breast cancer is marked by the breast lumps of greater than $5 \mathrm{~cm}$ in size and spread to the others parts of the body. If detected and treated in this stage, survival rate is as low as $21 \%$.

These survival rates [2], make it clean that it is extremely important to detect breast cancer in its earliest stage of development.

\subsubsection{Breast Exams}

There are three common methods used for detection of breast cancer: breast selfexamination (BSE), clinical breast examination (CBE) and mammography.

BSE is a method recommended for early detection of breast cancer. With previous knowledge and training in this method, every woman may examine herself at home. BSE should be performed once per month. In addition, every woman potentially could be the more familiar with her own breast tissue than a professional clinical examiner, making herself highly sensitive to subtle changes in her own breast tissue and thus more likely to detect changes in her own breasts.

A complete clinical breast examination (CBE) serves as an important complement to mammography. It is recommended that $\mathrm{CBE}$ be performed annually by professionally trained health care physicians. Compared with mammography, this methodology may be advantageous for young women. Young women usually have a more dense breast tissue, which is not conducive to good mammography images.

Mammography is x-ray screening of the breast tissue. "The American Cancer Society recommends women age 40-49, have a routine screening mammogram every 1-2 years, and those over the age of 50 have an annual screening mammogram" [3]].

Each of these breast examination methodologies has benefits and shortcomings. If all three are practiced according to guidelines, the optimum plan for the earliest detection is in effect. The problem with BSE and CBE is that more skill is required to detect relatively minor signs of early breast cancer. It has been found that only a small number 
of women perform BSE in a skilled, consistent, and proficient manner. In one study, two groups of women were tested on BSE skills. Correct detection of breast lumps was found in only $25 \%$ [4]. In 1991 a population based case-control study was conducted in order to examine relationship between BSE and occurrence of breast cancer. Results showed that self-described proficiency in the ten recommended BSE steps was generally low in both the experimented group (290 women with advanced breast cancer) and the matched control group (433 women)., with both groups reporting similar use of BSE in the year prior to the reference date. However, in the small group of women in the study who reported more thorough $\mathrm{BSE}$, there was about 35\% decrease in the occurrence of advanced-stage breast cancer in comparison to those who did not completely perform BSE [ $\underline{5}]$.

It has also been noticed as well that a significant deficiency in skills and competency exists among health care professionals who perform $\mathrm{CBE}$. In the study of 80 physicians, the mean successful lump detection rate was only $44 \%$ []].

A recent study using data from the Canadian National Breast Screening Study-2 [7] compared breast cancer mortality in two groups of women aged 50-59 years; those receiving annual screening consisting of two-view mammography and physician examination of the breasts and those receiving only physician examination. Finding showed no significant difference in mortality between the two groups suggesting that adding annual mammography screening to physical examination had no impact on breast cancer mortality.

According to the previous studies, we may conclude that the training process that would give expertise in techniques of the physical breast examination (BE) is of vital and paramount importance.

\subsubsection{Development of BSE training program}

The goal of the BSE training program is to educate women and health care providers in sensory-motor skills necessary to conduct proficient BSE. The sensory component involves both palpation with the fleshy pads of the fingers and tactile stimulation with various levels of pressure. The motor component includes training skills 
for proper finger palpation and breast tissue examination. Specific skill components, all integral to a comprehensive examination include a systematic pattern of search; palpation topography discrimination training; and finally varying finger pressures while examining the breast tissue [8]. It was found that the search pattern improved detection accuracy only if a correct palpation technique and a correct finger motion were performed (Saunders, Neelakantan, Criswell, Bloom \& Pennypacker, 1982 as cited in Pinto \& Fuqua, 1991).

The flats (from fingertips to first joint) of the three middle fingers are used for the examination. During the breast examination, the whole breast tissue has to be covered: from the top to the bottom, from the collarbone to the bra line, side to side from the center of chest area to the armpit area; and from the surface of the breast tissue to deep along the chest wall. Trainee uses the flats of the fingers to perform palpations in dimesized circles while applying three pressure levels at each site: light, medium and deep.

Light palpation is just enough pressure to move the skin but not to disturb the breast tissue underneath. This palpation moves the skin and the blood vessels but not the tendons just under the skin. Medium palpation involves enough pressure to explore and find lumps in the middle of the breast tissue. Finally, a deep palpation requires enough pressure to reach the breast tissue along the rib cage. All three pressure levels have been classified to make trainee capable of detecting breast lumps that may appear at any level in the breast tissue. During the examination, the breast area has to be completely covered with all three levels of palpations at each palpation site, along the non-redundant search pattern.

Three search patterns have been advocated to ensure coverage of the entire breast area: the vertical strip (VS) pattern; the concentric circle (CC) pattern; and the spoke of the wheel pattern. In 1986, an important study showed that the vertical strip pattern provided higher efficiency in covering $64.4 \%$ of the breast area compared to the concentric circle pattern with only $38.9 \%$ coverage. Likewise the study showed that the vertical strip pattern was superior to the spoke of the wheel pattern $(67.9 \%$ and $44.7 \%$ respectively) in coverage of the breast tissue [9]. 
Thus, the vertical strip has been chosen as an examination pattern during the breast examination (Figure 1 ).

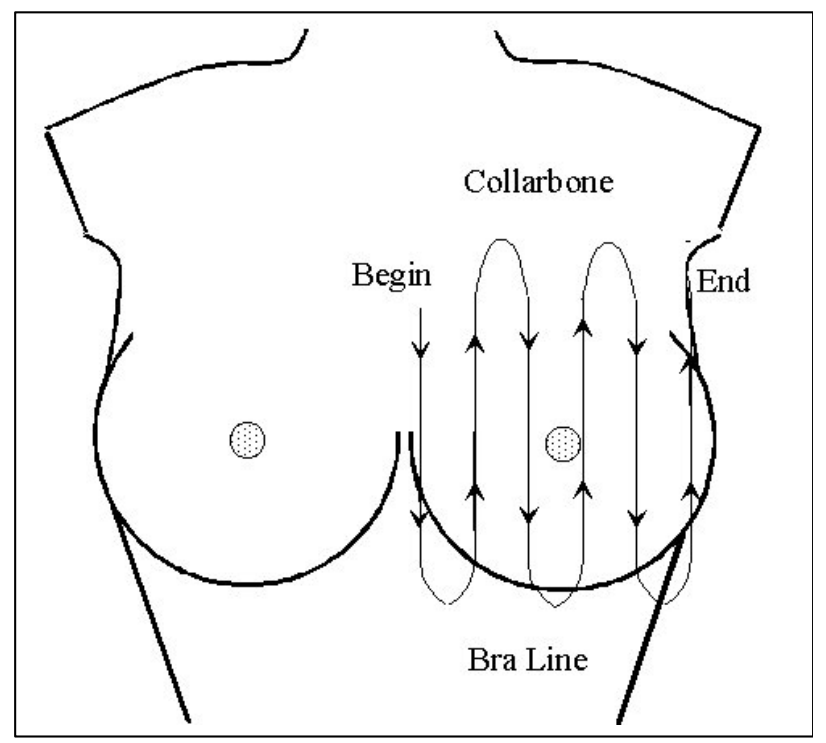

Figure 1. Diagram of Vertical Strip Examination Pattern

A silicon breast model is normally chosen for training and could become a true instrument with further development. Using just this model, a study has shown increasing success in correct detection of breast lumps (from $25 \%$ to $50 \%$ ) [4].

\subsection{History of previous work}

The initial idea of having an instrumented breast model for evaluating breast examination performance came from Dr. Susan Leight, Professor at West Virginia University School of Nursing. Having a set of sensors in the silicone breast model that would measure and record various levels of palpations as well as locate the place of applied palpation was the main goal.

With the help of engineering colleagues, an instrumented breast model was constructed consisting of eight linear voltage displacement transducers (LVDT). They were attached to the silicone model via Kevlar strings (Figure 2). Measuring 
displacement (as a function of palpation pressure applied to the model), data would be recorded during the exam and used for further analysis.

Midas data acquisition hardware and Labtech control software was used for collecting displacement data containing sensor displacement (inches) and time index (seconds). Data was saved in a file available for analysis and evaluation in Windows based applications such as Microsoft Excel and SPSS [1].

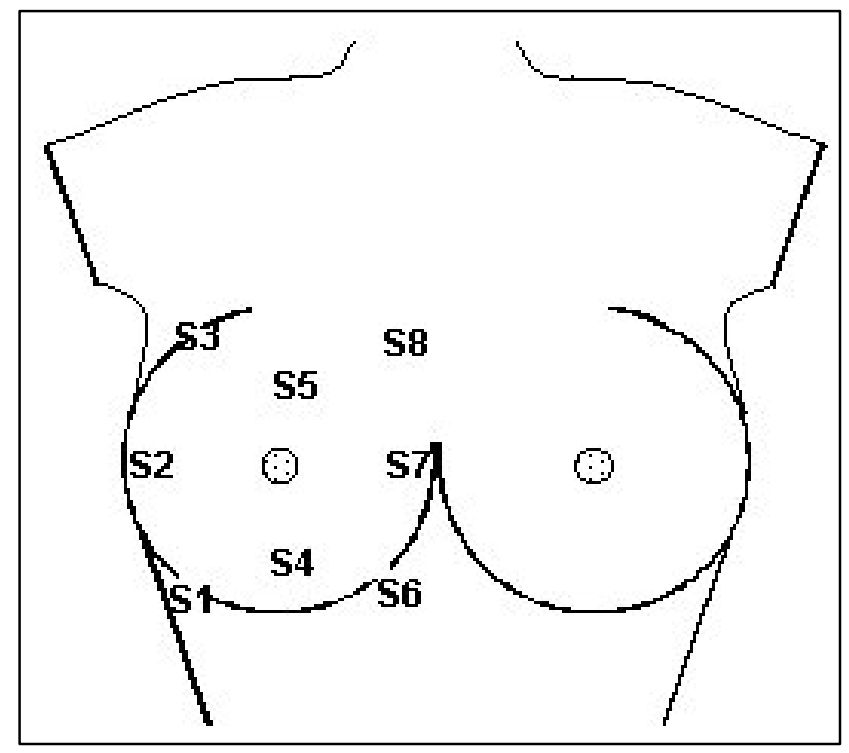

Figure 2 Diagram of Kevlar String Placements within the Breast Model

The training program included a pre-test performance on the model in order for baseline techniques to be established. It was followed by a video training presentation about discrimination of different levels of palpations and search pattern. The final examination was performed on the breast model in order to demonstrate to what extent the trainee had improved. The post-test results showed progress in overall performance with a longer time as an indicator of a thorough examination of the breast area and an increased depth of displacement in the model post-test demonstrations [1].

Analysis and performer's evaluation were based on output results in the form of a graphical time plots and each LVDT displacement (Figure $\underline{3}$ ). Although such graphic presentation gave useful information, it was very difficult to understand without in depth 
knowledge of the entire system. Correct interpretation of results required previous calibration and removal of negative offset from the plots.

Dr Leight pioneered the first automated examination model with biomedical evaluation of breast examination performance [1]. Previous work in this area has relied solely on observational measurement of trainee performance. This model clearly needed a more user-friendly display and more reliable and trouble free instrumented breast model.

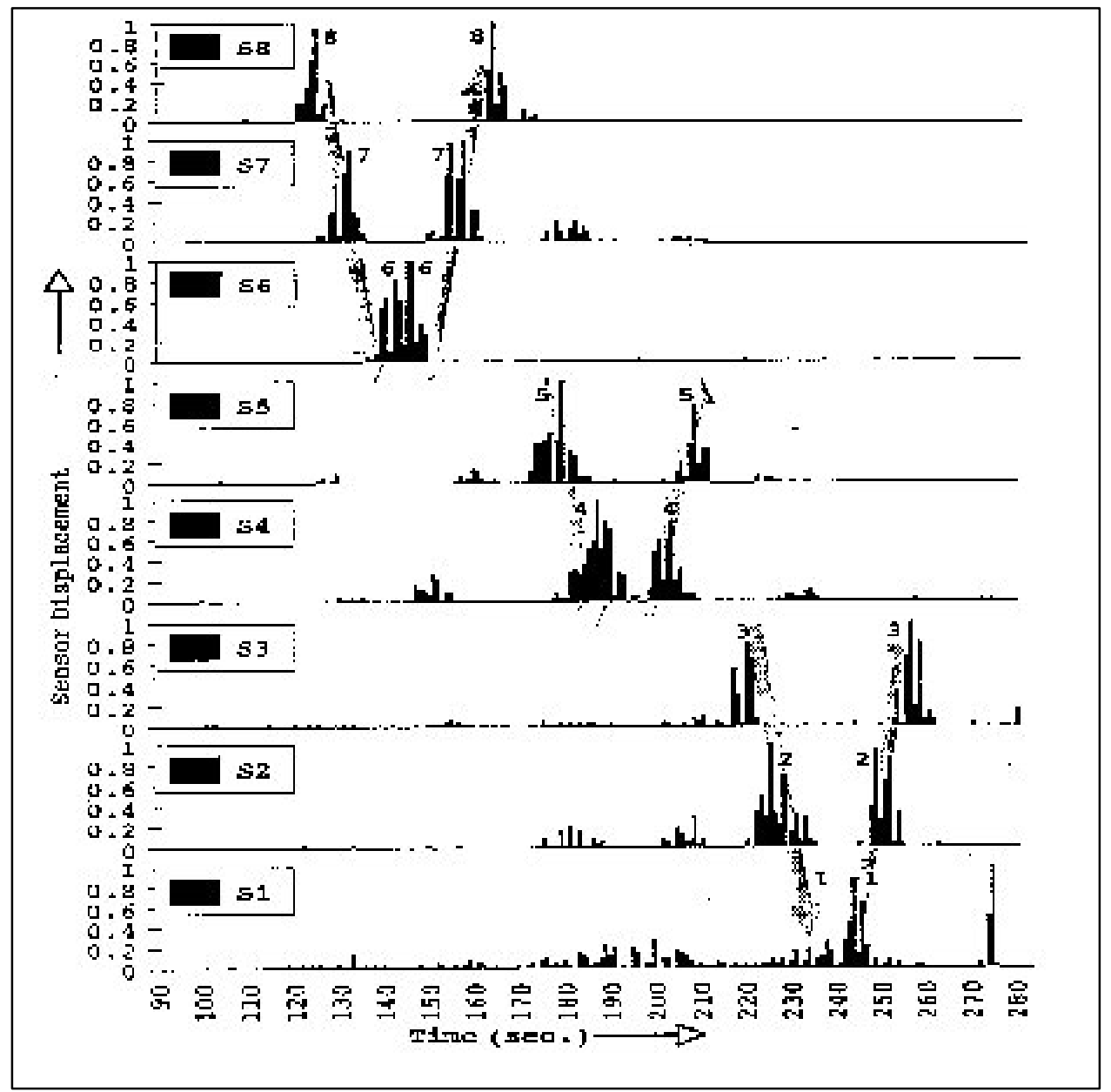

Figure 3 Original System Output [1]

A graduate student from West Virginia University, Ms. Christy Schmidt reengineered and upgraded Dr. Leight's instrumented model from a primary data collector into a self-contained interactive training system. Windows based software integrates 
training with multimedia presentation about the palpation techniques and search pattern. Brief tutorials about both breast cancer and the use of the application were included. Following the training sequence, the trainee can perform a manual examination on the instrumented model and receive a graphical performance feedback [10].

The main impact has been in the automation of the system and more understandable display of results from the instrumented model.

The exam evaluation algorithm used two developed fuzzy logic systems to determine finger position and palpation level from displacement data recorded into an Excel worksheet table. The final result is displayed on a summary Feedback Screen which include the following data: personal risk information; total time of examination; a graphic depiction of the trainee's examination compared with the expert's examination; an overall proficiency score; and an explanation of the results (Figure $\underline{4}$ ).

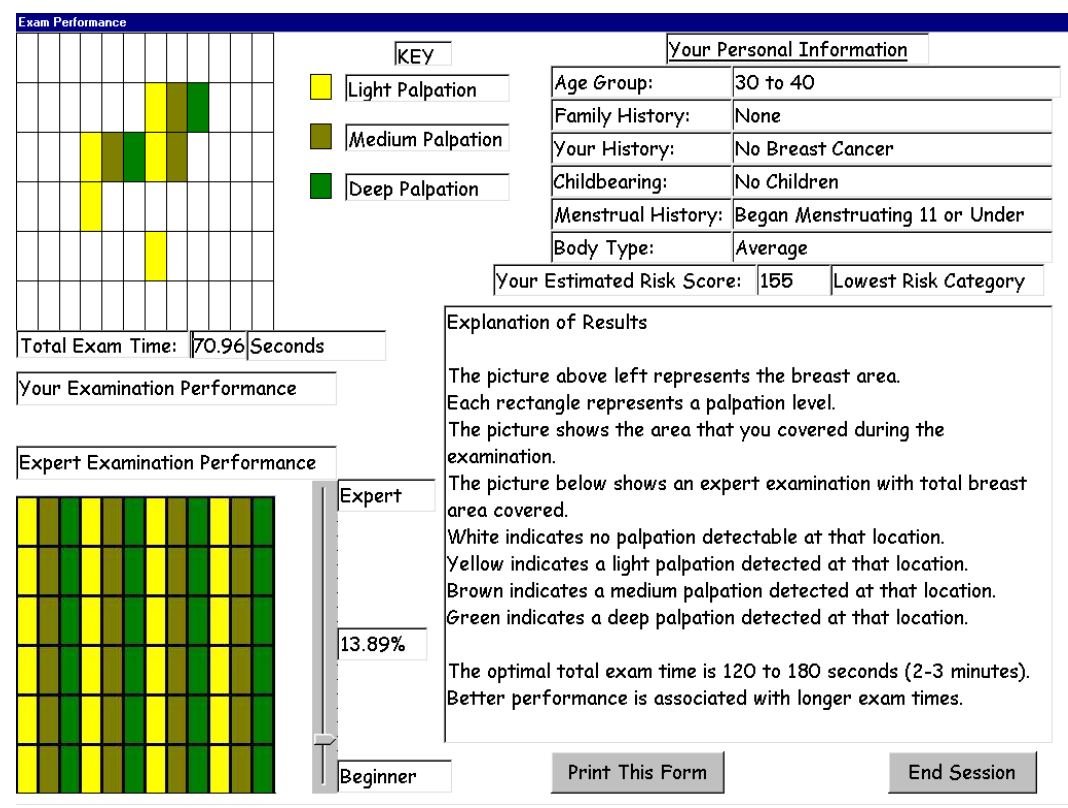

Figure 4 Example Feedback Screen [푸]

This project was named the Breast Examination Training Instrument (BETI) (Figure 5). The implemented technology fulfilled the stated goals of interactive education, of real-time examination, and of objective measuring of learner's performance. 
The cost of the LVDT prototype was less than $\$ 3000$ without the PC. Clearly, a more reliable, accurate and lower instrumented model was needed.

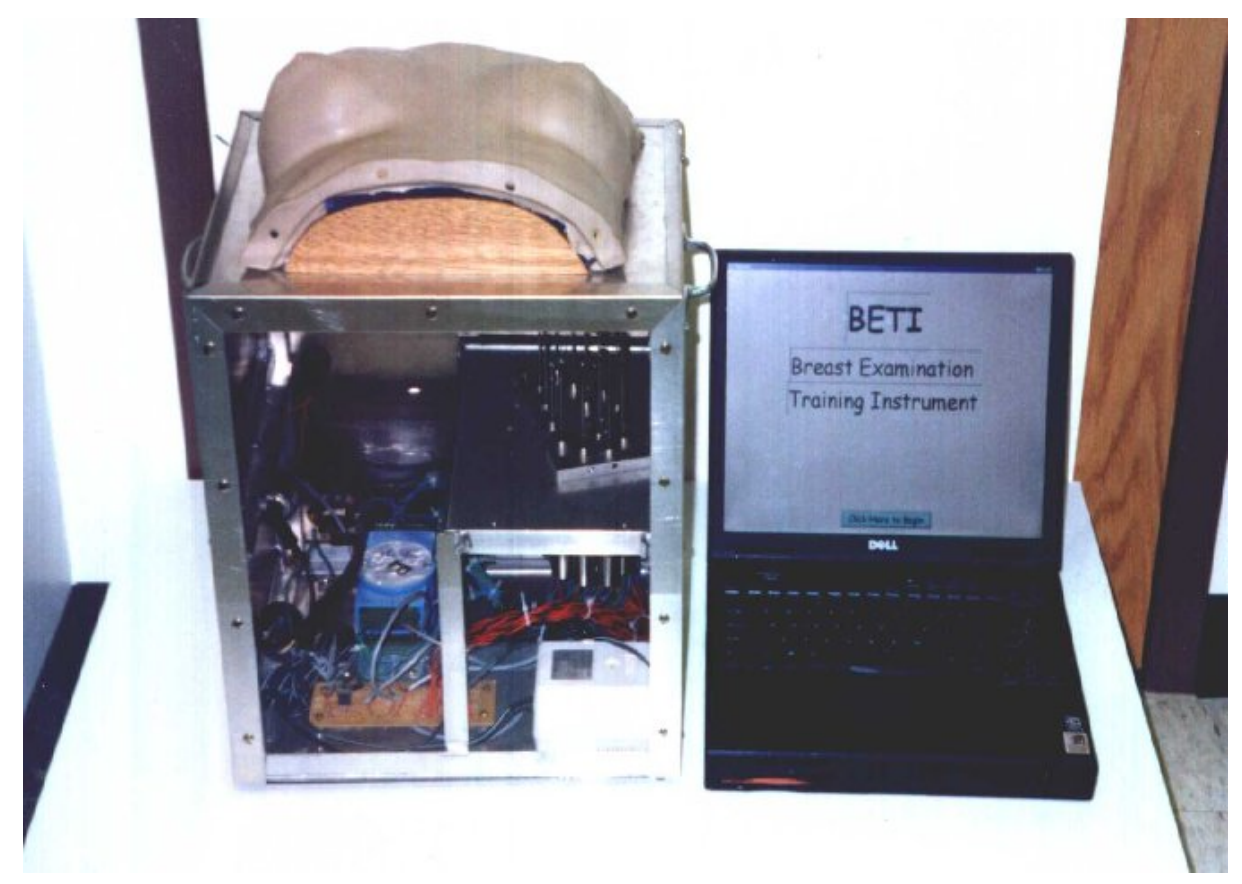

Figure 5 Prototype model of BETI $[\underline{\mathbf{1}}, \underline{\mathbf{1 0}}]$

\subsection{Work proposal}

In the search for advanced sensing materials, input requirements were:

- Touch sensitive - signals are generated when fingers touch the sensing material

- Pressure sensitive - the magnitude of the signal increases/decreases continuously according to applied pressure on the material

- Position sensitive - an X-Y location of the point of applied pressure can be determined

- Flexible - the material should be adjustable to the oval three-dimensional shape of the breast simulator

- Reproducible - the sensor pattern should be precisely duplicated in each unit

- Durable - the material should not quickly deteriorate under heavy use 
- Price - the sensing material needs to be inexpensive and correspond to one-third or less of the budget needed for prototype model

Requirements for the software development included:

- Interface with a new device

- Visualization of the real-time process (during the examination and training steps)

- Advanced interactive training procedure

As a full response to the projected input requirements, the author believes that the resulting system is the state of the art in new sensor technologies and a new standard in advanced educational and training instruments. The name of the project has not been changed, to honor the previous projects of Dr. Sue Leight [1] and Christy Schmidt M.S.E.E. [10], called the Breast Examination Training Instrument (BETI). It is the same abbreviation that will be used in further description of the system. 


\section{CHAPTER TWO}

\section{STATEMENT OF PROBLEM}

Work on this project is presented through the development of an improved version of the Breast Examination Training Instrument. This work is based on a previous prototype developed by Dr. Sue Leight and later improved by Ms. Christy Schmidt.

My work on this project included:

- Search for advanced sensors that would be used in an instrumented breast model. The sensor should be flexible and reliable with a time of use. The sensor should detect and locate palpation more precisely. Low cost was a serious consideration

- A development of automated BSE training software that would implement an interactive training process of BSE techniques described in 1.1.3., an examination of an instrumented breast model, and a non-subjective evaluation of the user's results from the examination

- A system integration of the breast model device and software that would produce a fully automated Windows application 


\section{CHAPTER THREE}

\section{SENSOR TECHNOLOGY}

\subsection{Sensor Specification}

Exploration of new sensor technologies is ongoing. Touch sensor specification include:

- A touch sensor should be a single-point contact within the sensory area (array, matrix, etc.). The size of the sensor would depend on the size of the sensory area and the resolution required for the application. The ideal sensor should be of size 1-2 $\mathrm{mm}^{2}$. This allows an array with a spatial resolution of 1-2 $\mathrm{mm}$.

- The ideal resolution of the sensory area should be one sensor per 1-2 $\mathrm{mm}^{2}$.

- The sensitivity of the touch sensor should cover a range of human palpation classified into three levels of pressure: light, medium and deep. The range 0.4 to $10 \mathrm{~N}$ was considered satisfactory for the requirements in the BETI application together with an allowance for any accidental mechanical overload.

- An optimal pad with all sensor bandwidth of $100 \mathrm{~Hz}$.

- The sensor's characteristics must be stable and repeatable with low hysteresis. A linear response was not necessary, as information processing techniques could be used to compensate for any moderate non-linearities.

- The touch sensor should be robust and protected from environmental damage

\subsection{Other Sensor Technologies}

Many physical principles have been explored and considered for the development of tactile sensors. In most cases, the development in tactile sensing technologies is application driven. A tactile sensor highly depends on the material used for the sensor. 


\subsubsection{Resistive based sensors}

Resistive based sensors are based on materials that have defined force resistance characteristics. The basic principle of this type of sensor is a measurement of the resistance of a conductive elastomer or foam between two points. The elastomer may consist of carbon-doped rubber (Figure $\underline{6}$ ).

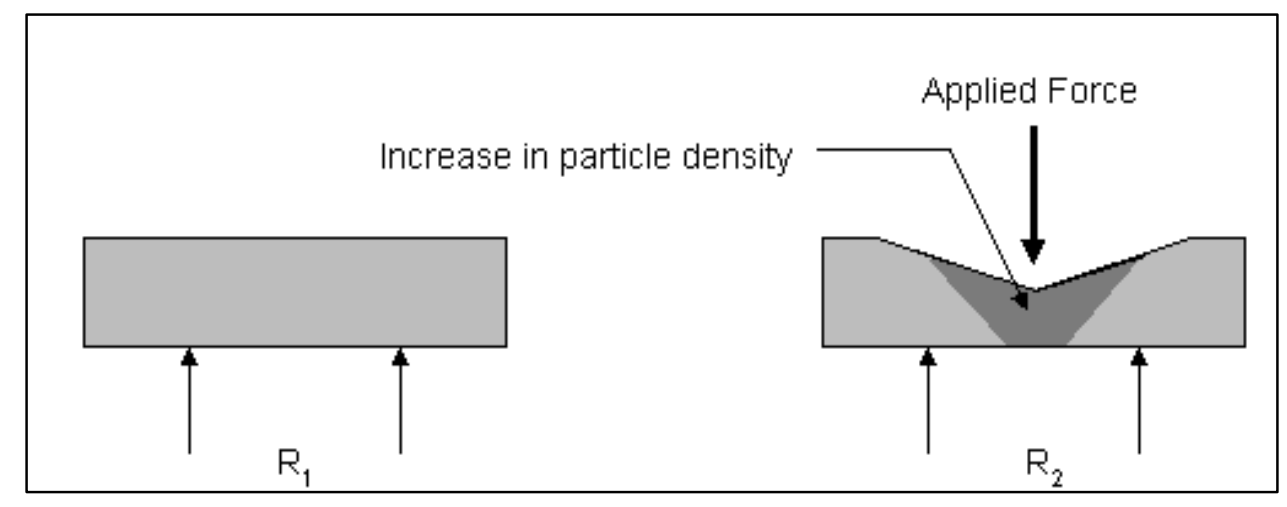

Figure 6 Basic principle of resistive based sensors

The resistance of the elastomer changes with the application of force, resulting in the deformation of the elastomer and an altering of the particle density. Using this type of sensor, measurement can be achieved either by using a dot-and-ring arrangement on the substrate or elastomer cords laid in a grid pattern. An advantage is an easy construction of a tactile image with a "good" resolution.

Yet, this type of sensor has some disadvantages. It has a long non-linear time constant. In addition, the time constant, when the force is applied, can be different from the time constant when the applied force is removed. Force-resistance characteristics are non-linear, requiring signal processing algorithms. The elastomer can become permanently deformed and fatigued, leading to permanent deformation. A resistive medium may migrate over a period of time. 


\subsubsection{Force Sensing Resistor}

A force sensing resistor is a conductive polymer, which changes resistance when force is applied on its surface. A sensor is presented as a polymer sheet, which has had the sensing film applied by screen printing. The sensing film consists of both electrically conducting and non-conducting particles suspended in a matrix. When a force is applied on the surface of the sensing film, it causes particles to touch the contacting electrodes, changing the resistance of the film. This type of sensor can be easily interfaced and operated (Figure 7).

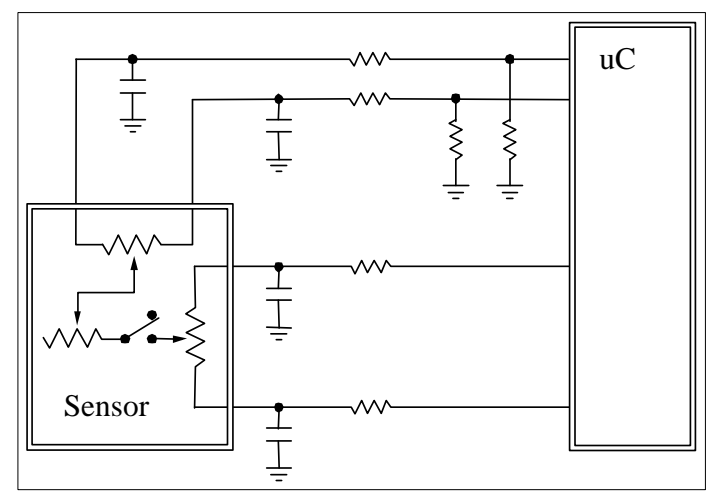

Figure 7 Basic circuit of the force sensing resistor [22]

\subsubsection{Piezoelectric Polymers}

Polymeric materials that exhibit piezoelectric properties are suitable for use as touch or tactile sensors. Polyvinildene fluoride (PVDF) is not piezoelectric in its raw state, but can be made piezoelectric by heating the PVDF within an electric field. PVDF can be 5 microns to $2 \mathrm{~mm}$ thick layer with metalization applied on both sides that are meant to collect the charges and permit electrical connections to be made. PVDF is well known for its high sensitivity, broad bandwidth and wide dynamic range. One of the most recent developments in piezo polymer technology is the piezo cable. The cable has the appearance of a standard coaxial cable, but it is made of a piezoelectric polymer insulator between the copper and braid, outside and inside the conductor (Figure $\underline{8}$ ). The cable 
features the same piezoelectric properties of piezo film sensors. This type of sensor is capable of sensing distant, small amplitude vibrations caused by rain or hail on even the impacts of heavy trucks. Experiments have shown linearity for increasing force. In the BETI application, a matrix of vertically and horizontally lined sensors would give precise location of the applied pressure.

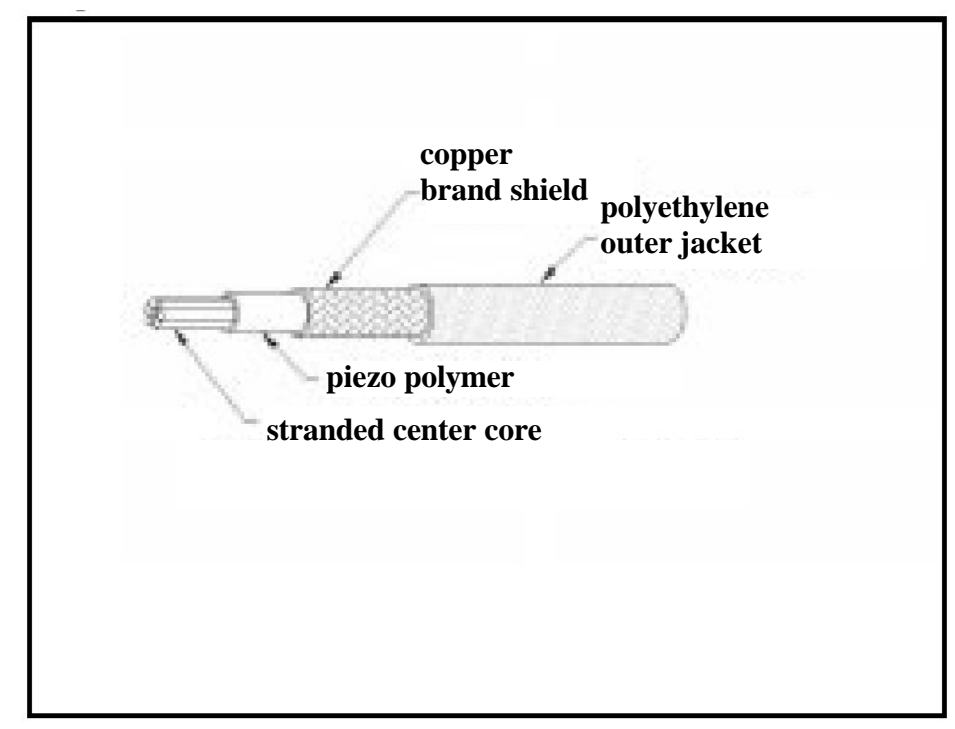

Figure 8 Structure of piezo cable [를

\subsubsection{Optical Sensors}

The expansion of the optical technology in the recent years has brought the development of wide range of tactile sensors. The basic principle is classified into two classes:

- Intrinsic, where the optical phase, intensity, or polarization of transmitted light are modulated without interrupting the optical path

- Extrinsic, where the physical stimulus interacts with the light external to the primary light path.

Some potential benefits of using optical sensors include:

- Immunity to external electromagnetic interference

- Intrinsically safe. 
- The use of optical fibre allows the sensor to be located some distance from the optical source and receiver.

- Low weight and volume.

Both optical sensors can be used for touch, and force sensing. However, extrinsic sensor would be better practical solution for the purpose of the BETI application. The principle of the extrinsic sensor is based on the intensity measurement. Therefore, easy construction and less optical processing would be required.

Photoelasticity is the phenomena where stress or strain causes changes in optical properties of the material. Using the optical transmitter, light is passed through the photoelastic material. When the force is applied, material is stressed causing the change in light polarization and hence the intensity of the light at the optical detector (Figure $\underline{9}$ ).

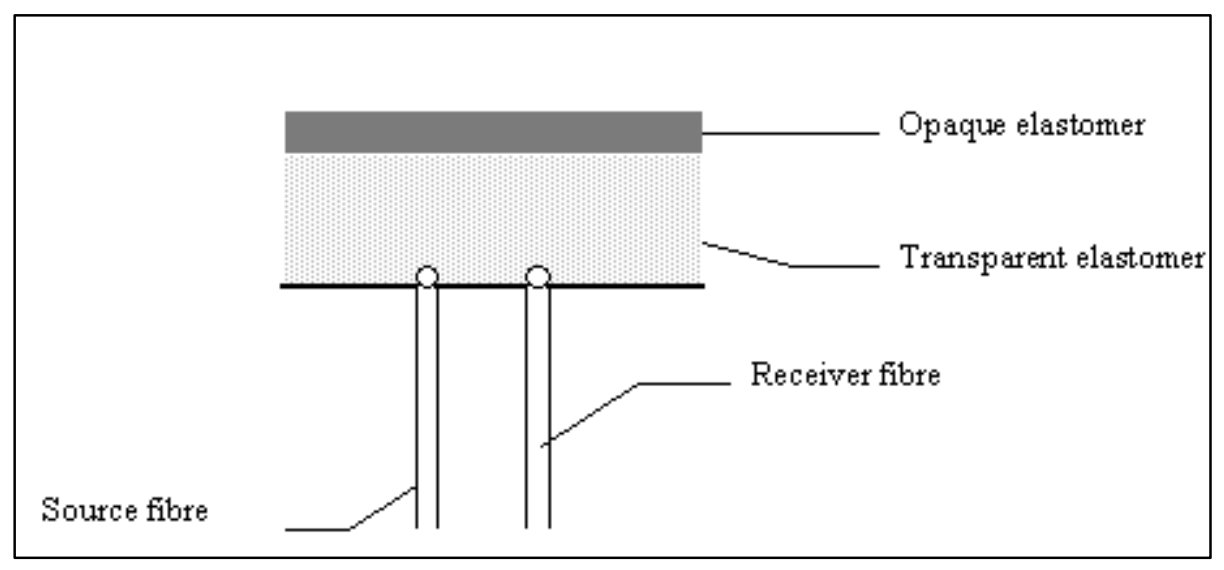

Figure 9 The principle of the optical sensor 


\section{CHAPTER FOUR}

\section{SYSTEM DESCRIPTION}

\subsection{Overview}

BETI is a sophisticated interactive instrument whose objective is to educate and train in the field of physical breast examination. The new hardware is based on a tactile sensor device attached to the standard silicon breast model and connected to the PC via a serial connection. The silicone breast model, from the Mammacare Company is designed to have an anatomically correct shape and a life-like feel. The tactile sensor device, a patented product of Tactex Corporation, is based on polymer foam (urethane, silicone, styrene) with an embedded matrix of opto-electronic transducers. Applied palpation on the silicone breast model causes deformation of the foam which changes optical properties. A proportional signal is generated in an embedded fiber optic or optoelectronic transducer. An electronic support receives the signals from all sensors and sends them to the PC through the serial connection. Figure $\underline{10}$ shows a general overview of the system.

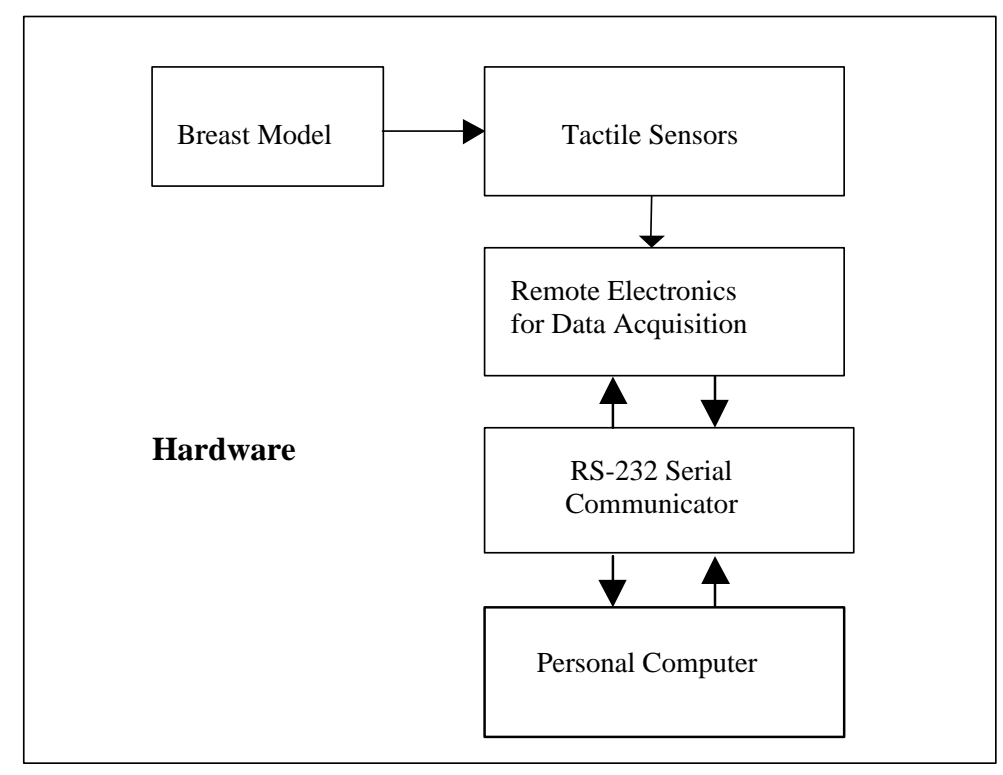

Figure 10 Hardware Schematic Description of BETI 
The software integrates six components: a graphical user interface (GUI), a calibration algorithm, an interactive instructional video, a real-time data collection component, an exam result evaluator, and interpretation and a result presentor. During the real-time data collection, the program communicates with the sensors device, recalling the current values of the signals from all opto-sensors within the pad sheet matrix. The received data is filtered, scaled and stored in the tables of coverage. A real-time algorithm then calculates the centered point of applied palpation on the breast model and records it. The software is written in $\mathrm{C}++$ while the GUI is constructed under the Borland $\mathrm{C}++$ Builder 5. Rational Rose for C++ was used for Object Oriented Analysis and Design of the application. Figure 11 presents a general diagram of the system.

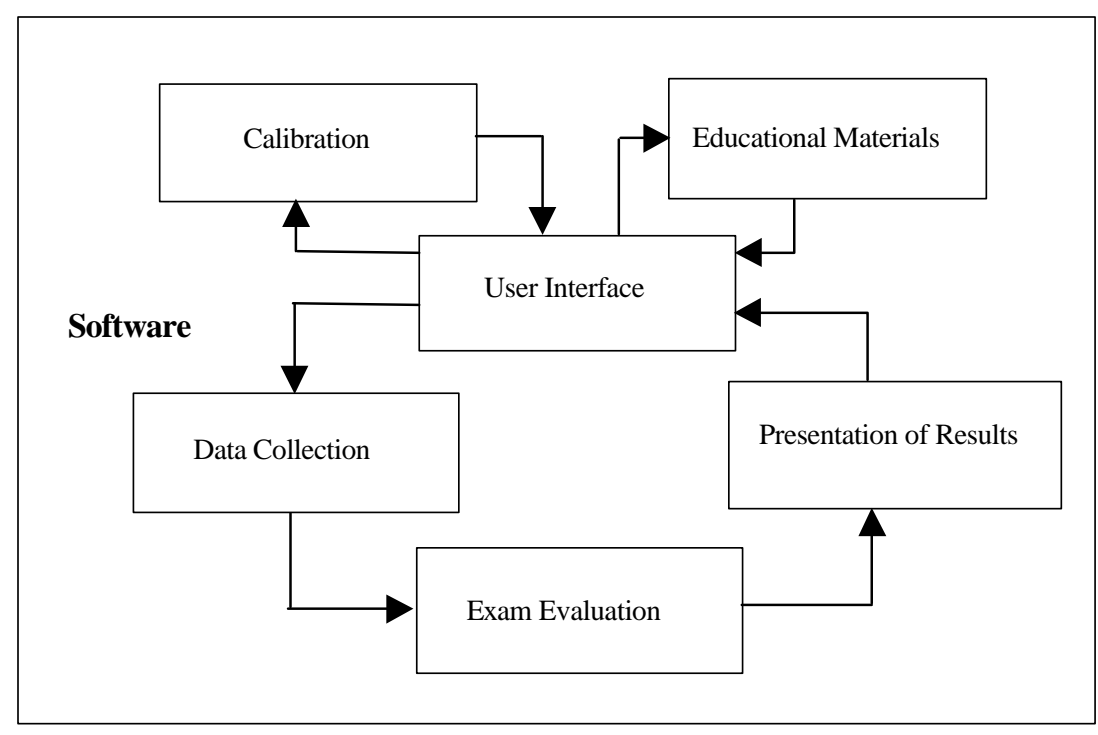

Figure 11 Schematic Diagram of the Software 


\subsection{Detailed Hardware Design}

\subsubsection{The Silicone Breast Model}

The hardware is made of a silicone breast model (SBM), attached to a tactile sensor device. The silicone model is filled and molded in order to give an impression and a physical sensation that are as close as possible to nature during the examination. There are several models of SBM with different properties regarding the size, texture and implanted simulated lumps. The texture of the model is important because all breast tissue has a texture called nodularity. Women may have different breast tissue nodularity. Breast tissue may feel like grain or sand under the skin. Artificial breast lumps are implanted in the SBM to be used during the training process. They are placed in different locations and on different levels in the model so that the user can locate them using the different levels of palpation. Breast lumps may be located anywhere in the breast: close to the surface, down to the ribcage or anywhere in between.

\subsubsection{The Touch Sensor}

The tactile sensor pad, a product of Tactex Company, is placed underneath the SBM. The sensor sheet is based on deformable polymer foam, covered with protective membrane (Figure 12). Polymer foam contains an embedded matrix of sensors. Each sensor works on the principle of deformation of an 'optical integrating cavity' [11]. This principle simulates the method of detecting pressure / displacement. Any applied palpation on the SBM is registered as a pressure that deforms the foam. Deformation of the foam affects the changes in optical properties of the foam. A simple optical transducer can sense the change (Figure 12). A fiber optic brings the generated signal from the optical LED to the local area of the foam. This turns the local area of the foam into an illuminated cavity. Deformation of this cavity affects the proportional change of illumination energy intensity. Another fiber optic, receives the optical signal and sends it to a photodiode. The change in the illumination energy intensity is proportional to the 
level of electrical signal generated from the output of the photodiode. This is how the change is detected and measured.

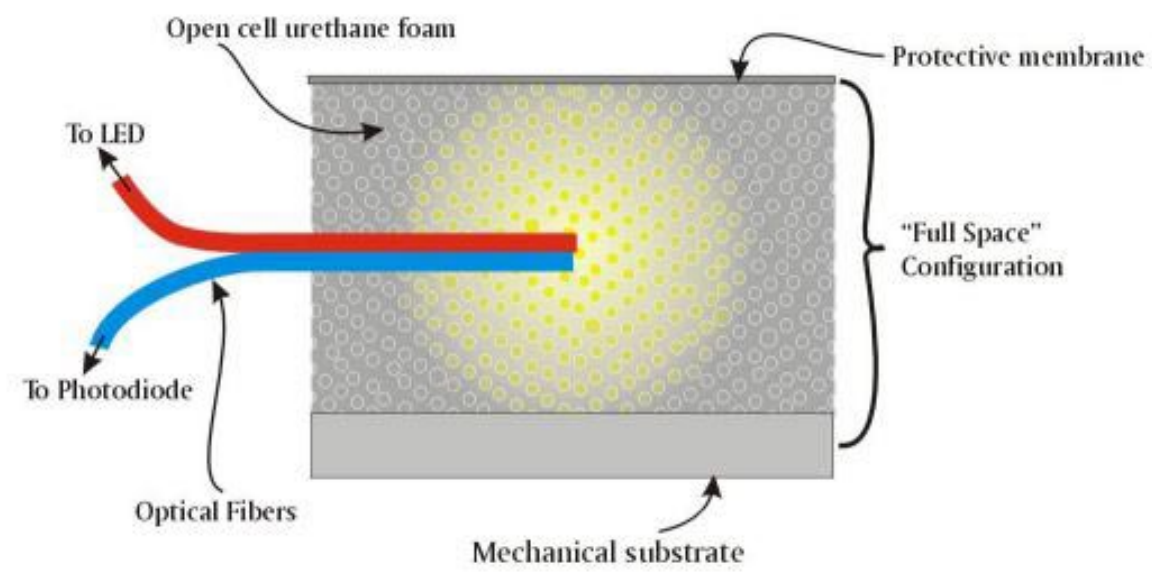

Figure 12 The KINOTEX ${ }^{\mathrm{TM}}$ sensor [11]

The polymer foam has dominant properties of isotropic scattering medium. Any illuminated energy, from the input fiber will be scattered and partially absorbed by the foam material. This would cause the illumination to be seen like a halo. The intensity of the given energy depends on the distance from the source, the scattering and absorption properties of the medium. A characteristic scattering length $(\mathrm{Csl})$ represents the integration of the above mentioned properties $\left[\mathrm{KINOTEX}^{\mathrm{TM}}\right]$. The scattering energy can therefore be expressed as:

$\boldsymbol{E}_{\boldsymbol{s}}=\boldsymbol{E}_{\boldsymbol{o}}\left[\boldsymbol{1}-\boldsymbol{e}^{-t / C S L}\right]$, where $\mathrm{E}_{\mathrm{s}}$ is the scattered energy and L is the distance traveled. $\mathrm{t} / \mathrm{C}_{\mathrm{SL}}$ represents a normalized dimension, the optical thickness or optical depth.

although thickness $\mathrm{t}$ and Csl are proportionally reduced (Figure $\underline{13}$

is placed inside the optical depth Csl, the detector would see the integrated intensity of scattered energy in its vicinity. Integration is considered as a volume around the detector

cavity around the source or detector. When Csl is reduced, the integrating cavity shrinks 
as well, causing scattered energy to increase inside the cavity. If the energy detector is placed a distance from the source of less than optical depth Csl, it will record an increase of energy [11].

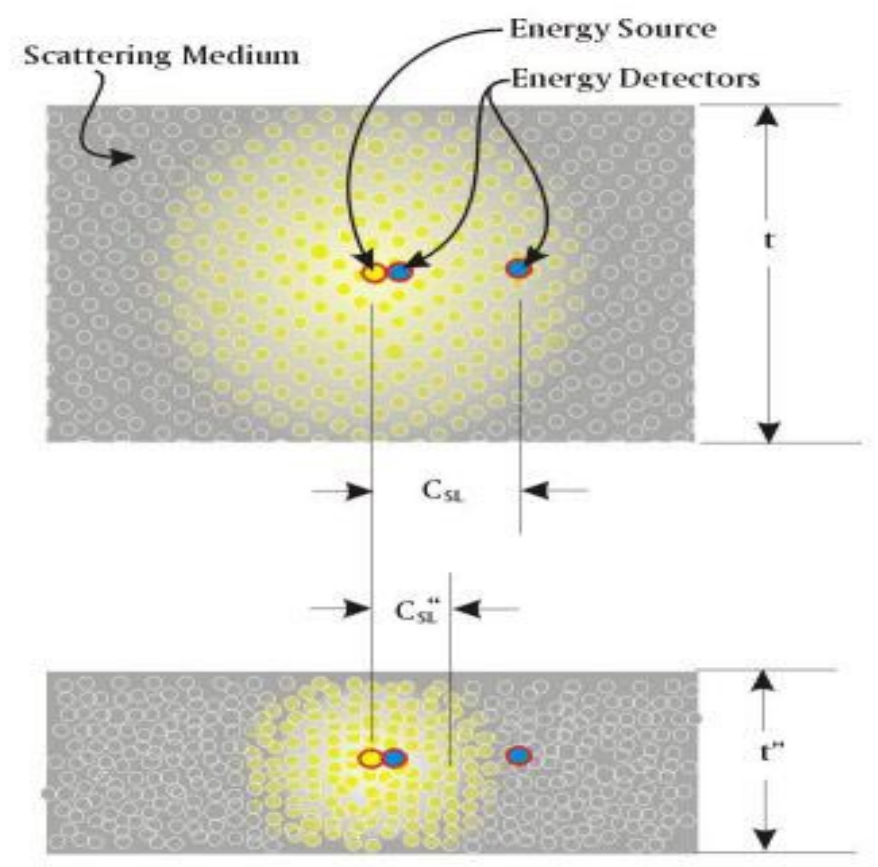

Figure 13 KINOTEX $^{\mathrm{TM}}$ sensor principle [11]

The energy detector is appropriately placed so that it can not detect any energy, radiated directly from the source but only a scattered energy. On the other side, if the detector is located on a distance greater than Csl, it will detect decrease of energy. In the matrix of sources and detectors, an adequate spacing should prevent any energy interference between cavities.

The characteristics of TACTEX sensors used in the BETI project are of a crucial importance for its efficiency. The relation between the displacement and the output signal and the relation between load-pressure and output signal should be considered. The experimental results have shown linearity with respect to the deflection of the foam material (Figure 14). The sensors can resolve relative changes as small as $0.1 \%$ of total 
thickness but with higher pressure, accuracy decreases due to the visco-elastic properties

15). Raw data may be obtained and used for further experiments in linear modeling.

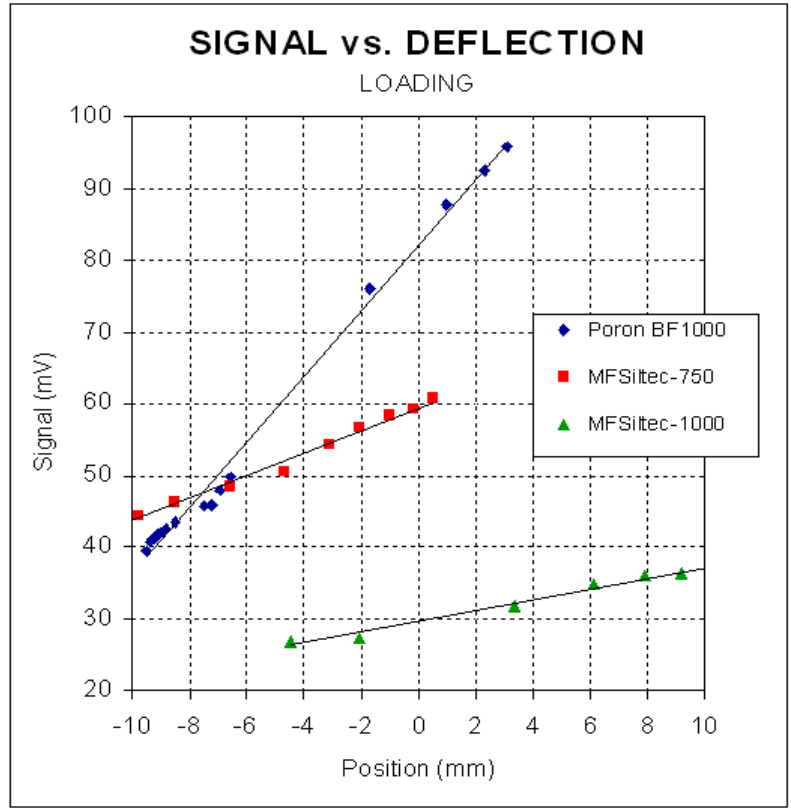

Figure 14 Deflection vs. Signal of KINOTEX sensor [11]

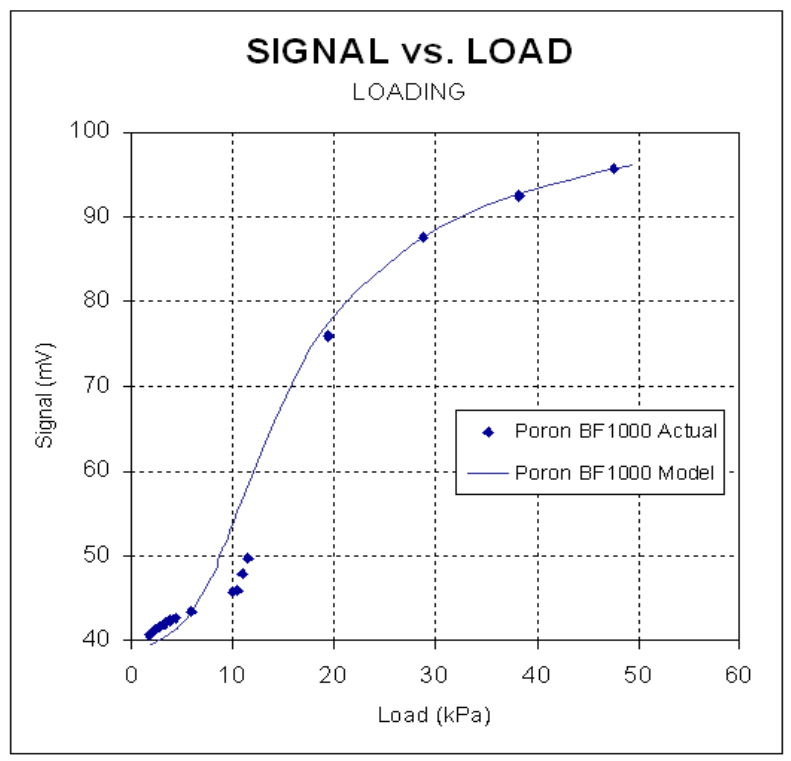

15 
The device is capable of sensing pressures from as low as $0.1 \mathrm{kPa}(.01 \mathrm{psi})$ to over 200 $\mathrm{kPa}$ (25 psi) and sensing deformations as small as $0.025 \mathrm{~mm}$ (.001") to many $\mathrm{mm} /$ inches 11].

Sensors are arranged in a regular matrix. They are coupled to remote electronics via optical fibers. Fibers are chemically inert and immune to EMI. The signals from the communicates with the outside world through an RS-232 communication link. A set of API functions has been developed by TACTEX Company [_]. They have been used in a further software development. Figure $\underline{16}$ is a graphic representation of the device.

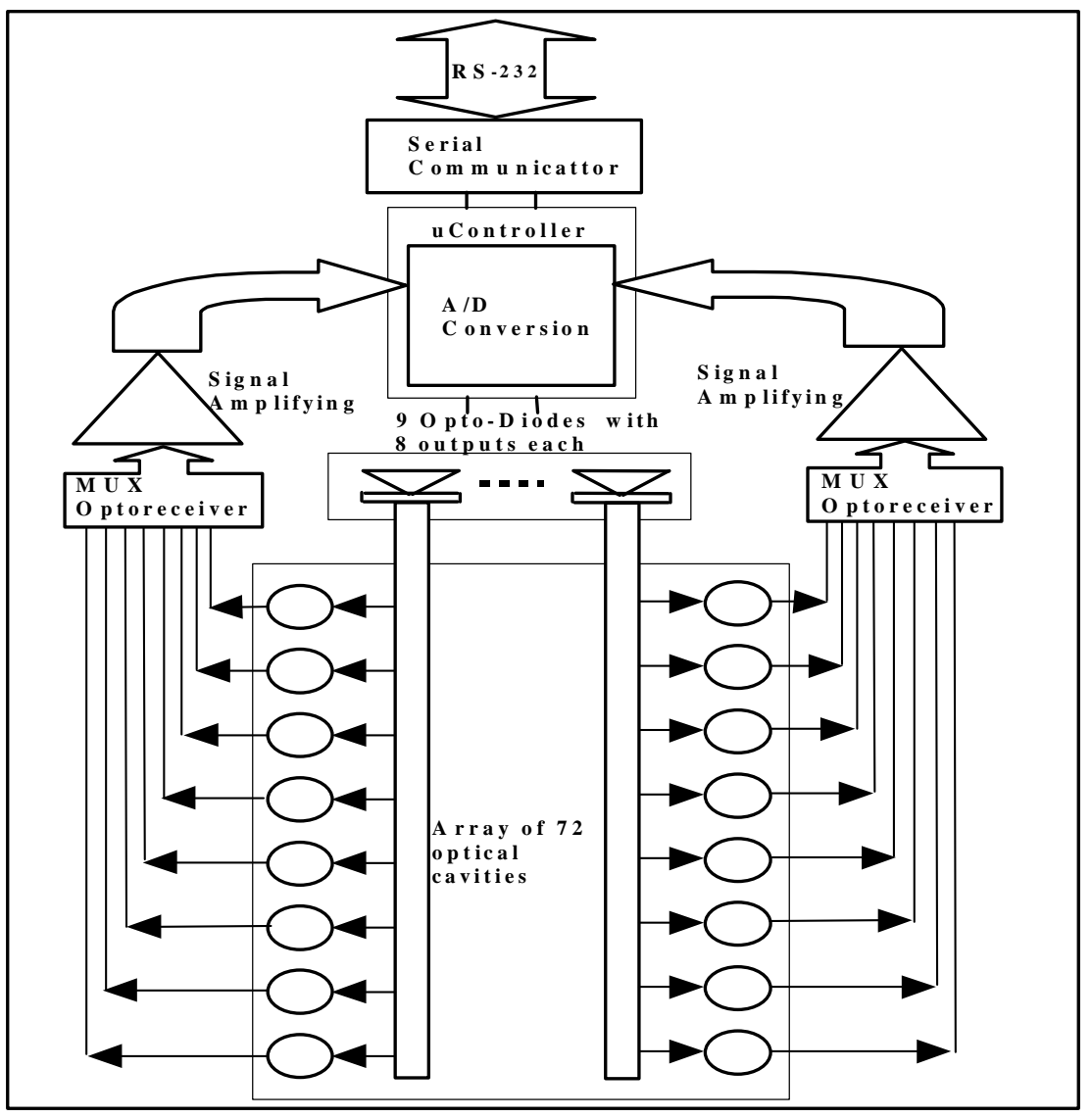

Figure 16 Schematic of the TACTEX device 


\subsection{Detailed Software Design}

The value of BETI lies in its being an easy-to use method. A carefully designed Graphic User Interface (GUI), developed in Borland C++ Builder 5 [14], allows the user to easily operate BETI. The application can be used by any person who can read and who can operate a computer mouse. Places where the user input is required are arranged in the form of multiple choice questions. All six windows are in the graphic mode 800x600 pixels and higher. Each page contains a help button that gives detailed instructions about the displayed form and prospective options for the user. Information is limited and presented slowly to eliminate any possible confusion and to clearly describe the current state of the machine.

\subsubsection{GUI}

All training components are incorporated into one software package. A training video is presented through a convenient display with a user control form. In addition, the video presentation is interactive so that the user can learn the examination techniques simultaneously while watching the video and practicing on the model. The GUI can show feedback from the model as the exercise is performed and may instruct the user in corrections or improvements during the practice exercise. This component makes BETI a highly sophisticated instrument with a fully automated educational process. The user interface is the main link between the PC and the user in terms of calibration, personal estimator survey, real-time data collection and final evaluation stage.

\subsubsection{Educational Materials}

Since the main purpose of BETI is to educate women and health care physicians about the techniques in physical breast examination and breast cancer, two approaches have been used to fulfill the goal: an interactive training video and a Power Point Tutorial through the Component Object Modeling (COM) server.

\subsubsection{The Training Video}

The training video has been developed and produced by the Mammacare Company [12]. The video includes theory and practice on manual breast examination. It 
follows seven main topics:

1. "Introduction";

2. "Clear model examination";

3. "Opaque Model Examination";

4. "Verification of Results";

5. "Examination of Boundaries";

6. "Breast Self Exam";

7. "Conclusion"

Each topic is linked with one pushbutton on the video menu on the computer screen. Buttons are numbered according to the sequence of the topics in the video material. After selecting any topic from the video menu, the user is forwarded to the video player form. The video player uses Windows Multimedia Player with video control features. The user is free to play, stop, pause or forward / rewind the video at any time.

There are two silicon breast models used in the training video: one is a clear model and the other one is an opaque model. The "Clear model examination" topic teaches users how to use their fingers, perform palpation and differentiate between the three levels of pressure (light, medium and deep) during the examination. The model is divided in two halves. Each half has a different texture-nodularity simulating various breast tissues. This model teaches a user that breast tissue may feel for example like grain or sand under the skin. While practicing palpation, the user is prompted to feel the difference in nodularity between the two halves. Artificial lumps are implanted in different locations of the silicone model. There are both hard and soft lumps. While applying different levels of pressure, the user can detect the lumps located close to the surface, in the middle, or at the bottom of the model. The video material carefully describes light, medium and deep palpation. Between each segment, the user is prompted to practice on the silicone model on the computer pad. The user then gets a response from the touch pad through a graphic on the computer display. The status of each touch sensor 
on the pad is displayed in one of four possible colors. Table $\underline{1}$ gives a description of applied pressure according to the displayed color.

\begin{tabular}{|l|l|l|l|l|}
\hline Displayed Color & Black & Green & Yellow & Red \\
\hline Applied pressure & No pressure & Light & Medium & Deep \\
\hline
\end{tabular}

Table 1 Description of applied pressure on the touch pad according to colors

The "Opaque model exam" topic on the video presents the search pattern during the breast examination. A straight vertical strip has been described previously in this text as the most successful approach regarding the percentage of covered area and the number of identified lumps. The user is advised to start with her / his palpation at the far corner of the model at the top (at the hollow of the armpit area). The user should continue moving the pads of the fingers straight down the side until the leading finger goes off the edge of the model. Then, the user should move over one finger width with short overlap slightly over the vertical strip. The user should continue the exam upward now to the top of the model until the leading finger goes off the top edge of the model. The user should then continue the same pattern over the entire surface of the model. The examination is done when the opposite corner from the starting one is reached. During the examination, the user has to use three middle fingers for palpations, use pads of fingers and make three small circles at each spot using light, medium and deep pressure. In the video instruction, wherever the user feels a lump, a sticker should be placed. Placed stickers on the breast model are used following the exam for verification of the examination results. When the user is done with the second topic, an examination on the instrumented model should be performed. At this point, the author was not in position to develop this training step as an automated application. However, in the future, a search pattern agent could be developed as well as detector of lumps that would evaluate success in number of correct lump detections. The author hopes that additional training measures will be developed in a near future and become integrated in the final version. 
The first two video topics covered three main components of the manual breast examination: palpation, three levels of pressure (light, medium, deep) and search pattern on the breast area.

The section of the video entitled "Verification of results" deals with the number of successfully identified lumps and the search pattern. The silicone model has to be physically turned over so that the user should can identify how many of the five initial lumps, she / he has been able to identify during the examination. If a placed sticker corresponds to an actual location of the artificial lump, identification is determined as successful. In addition, this topic presents some suggestions for further improvement (pace, slight overlap between adjacent vertical strips, three small circles at each spot with increasing pressure).

The "Boundary exam" topic of the video tells the user more about boundaries of the breast area. This topic covers the last two keys of the manual breast examination: perimeter and positions.

The final topic of the video, "Breast Examination" demonstrates all five components of the manual breast examination on a live model.

This video material is converted into Windows Media Video file and included in the latest version of the BETI.

\subsubsection{Tutorial}

The original tutorial was written by Dr. Sue Leight to educate users about breast cancer, breast examinations and use of BETI application. The new tutorial has been reformatted as a MS Power Point presentation. The tutorial is divided into three sections: information on breast cancer and detection methods, techniques and search pattern used in breast examination, and the user's manual for use of the BETI application.

The first section of the tutorial gives information regarding breast cancer and methods used for detection of the disease. The development of breast cancer is described through three stages of breast lump progression (localized, regional and distant). The risk factors for developing breast cancer are presented in order to show the importance of breast examination in early detection. Three methods are used in early detection of breast 
cancer: Mammography, CBE and BSE. Each method is discussed and screening recommendations are given.

The second section presents examination techniques and search pattern on the computer based breast model. Examination techniques include: correct use of fingers for palpations, three palpation levels at each site and description of the vertical strip search pattern as the most effective one in the breast examination. This section ends with an overview of all five components in the breast examination.

The last section of the tutorial explains how to operate the BETI application. The procedure used in the application is described through the available options in each form of the GUI. Careful explanation about the presentation of results from the model examination is provided.

\subsubsection{Data Collection}

Data Collection is the software component of the BETI application. The data collection component is divided into two parts: breast cancer risk estimator and online data acquisition from the touch pad.

\subsubsection{Breast Cancer Risk Estimator}

Dr. Leight's risk estimator is based on user's response from a given set of questions concerning the breast cancer. The risk estimator was added to BETI to make the user aware of the significance of breast cancer. The information for the risk estimator component was taken from a chart on a Health EdCo tri-fold display entitled "Caring for your breasts" [15]. The main factors that can increase the risk of developing breast cancer are a personal history, menstrual history, family history, age group and a body type. Each factor is formatted as a question followed by a list of optional answers. The user enters personal information by choosing and clicking the appropriate option button (optional answer) through the series of six multiple-choice questions.

The risk estimator evaluates the user's risk group, scoring answers to questions based on the above factors. Based on this score, the user is ranked into one of the four lifetime risk groups concerning the development of breast cancer: lowest, medium, high 
or highest risk group. Tables $\underline{2}$ and $\underline{3}$ show details about the scoring system of risk estimator.

\begin{tabular}{|l|l|l|}
\hline RISK FACTOR & CATEGORY & SCORE \\
\hline \multirow{5}{*}{ AGE } & Under 30 & 10 \\
\cline { 2 - 3 } & 31 to 40 & 30 \\
\cline { 2 - 3 } & 41 to 50 & 75 \\
\cline { 2 - 3 } & 51 to 60 & 100 \\
\cline { 2 - 3 } & Over 60 & 125 \\
\hline \multirow{5}{*}{ FAMILY HISTORY } & None & 0 \\
\cline { 2 - 3 } & 1 Aunt and Grandmother & 50 \\
\cline { 2 - 3 } & Mother or Sister & 100 \\
\cline { 2 - 3 } & Mother and Sister & 150 \\
\cline { 2 - 3 } & Mother and 2 Sisters & 200 \\
\hline YOUR HISTORY & No Breast Cancer & 0 \\
\cline { 2 - 3 } & Previous Breast Cancer & 300 \\
\hline \multirow{3}{*}{ CHILDBEARING } & First Baby Before Age 30 & 0 \\
\cline { 2 - 3 } & First Baby After Age 30 & 25 \\
\cline { 2 - 3 } & No Baby & 50 \\
\hline \multirow{3}{*}{ MENSTRUAL HISTORY } & Began Menstruating 15 or Over & 15 \\
\cline { 2 - 3 } & Began Menstruating 12 to 14 & 25 \\
\cline { 2 - 3 } & Began Menstruating 11 or Under & 50 \\
\hline \multirow{3}{*}{ BODY TYPE } & Slender & 15 \\
\cline { 2 - 3 } & Average & 25 \\
\cline { 2 - 3 } & Overweight & 50 \\
\hline
\end{tabular}

Table 2 Scoring system of the Breast Cancer Risk Estimator

\begin{tabular}{|c|c|}
\hline IF YOUR SCORE IS & YOUR CATEGORY IS \\
\hline Under 200 & Lowest Risk \\
\hline 201 to 300 & Medium Risk \\
\hline 301 to 400 & High Risk \\
\hline Over 400 & Highest Risk \\
\hline
\end{tabular}

Table 3 Evaluating system based on answers from multiple-choice questions

Answers are recorded and presented in the final presentation of results. MS Excel worksheet table (Figure 17) is initiated and filled with user's personal data based on the 
answers from the above questions. This table is used for an analysis of the user's performance during the examination. After answering all multiple-choice questions regarding the Risk Estimator, the user can start a real-time examination of the breast model. This is the second part of data collection component called Online Data Acquisition.

\begin{tabular}{|l|l|}
\hline Personal Info & \\
\hline Age & Under 30 \\
\hline Fam ily History & None \\
\hline Your History & No Breast Cancer \\
\hline Child Brearing & No Children \\
\hline Menstrual History & Began Menstruating 15 or Over \\
\hline Body Type & Average \\
\hline & \\
\hline
\end{tabular}

Figure Personal Information recorded in the worksheet table

Online data collection is performed during the user's examination on the instrumented breast model. While the user performs palpations on the breast model, the Using A/D conversion, those signals are converged into digital data. A set of API functions has been developed by Tactex for use with their touch pad. Reading data from reading raw data.

Reading raw data brings converged numeric data directly from the touch sensors.

Using new data from the pad requires additional normalization to be determined.

Reading normalized data however, is based on predefined normalization of raw

the calibration of the device. This data reading gives a linear function between applied load-pressure and converged signal. During the use of the touch pad, visco-elastic at regular time intervals. 
Reading normalized data from the touch sensors gives the converged values in a range 0 - 1023. For the purpose of this application, three levels of pressure have to be differentiated. Therefore, the range of numeric normalized data has to be divided into three sub-ranges that should represent the level of applied pressure. Table $\underline{4}$ describes the level of applied pressure on the touch pad, presented through the range of numeric values.

\begin{tabular}{|l|l|l|l|l|}
\hline $\begin{array}{l}\text { Applied } \\
\text { Pressure }\end{array}$ & No Pressure & Light Pressure & $\begin{array}{l}\text { Medium } \\
\text { Pressure }\end{array}$ & Deep Pressure \\
\hline $\begin{array}{l}\text { Normalized } \\
\text { Data }\end{array}$ & $<70$ & {$[71,300]$} & {$[301,700]$} & {$[700,1023]$} \\
\hline
\end{tabular}

Table 4 Applied touch pressure represented through the range of numeric data

The instrumented breast model is configured with the silicone breast model, attached to the touch pad device. The same applied pressure on different locations on the silicone breast model does not register equal readings. This is because of the three dimensional oval shape of the silicone breast model. Because the central portion of the breast is thicker, greater displacement must occur. Therefore, the scaling factor is defined for each sensor location in the touch pad. Experiments were done on the available used silicone breast model to define scaling factors-weights as shown in Table $\underline{5}$. These weights were used in the experiments described.

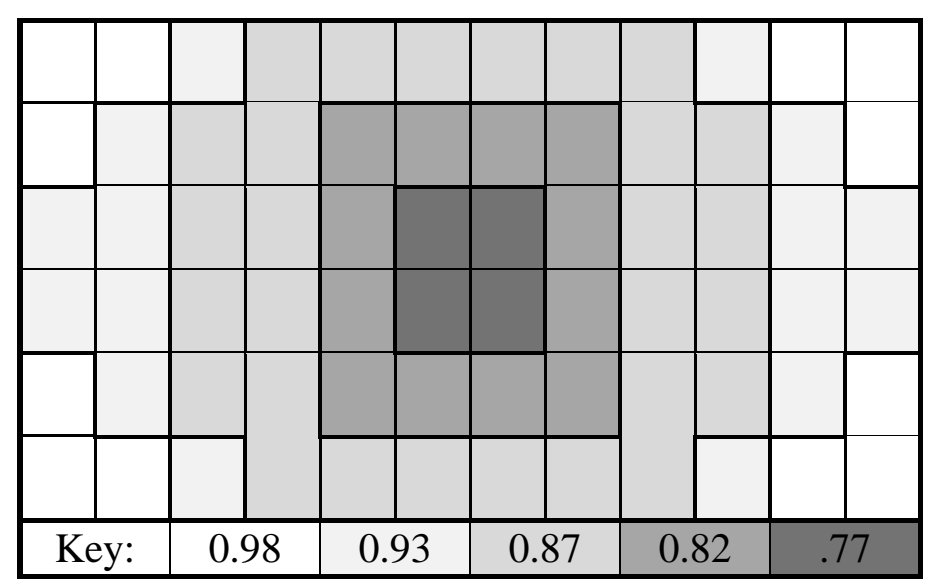

Table 5 Table of weights for 72 sensors of the touch pad 
Different silicone models may have different visco-elastic properties. Different shape and thickness' may also occur with other silicone models. A table of weights needs to be defined for each silicone model that is used in BETI. Configuration and calibrations of the weights is described in this chapter.

Data is collected every $50 \mathrm{~ms}(20 \mathrm{~Hz}$ reading rate). Data is filtered. For each sensor, there is a table of four last readings. Using LIFO buffer, when a new reading appears, the oldest one is removed from the list. Mean value is calculated and then classified into one of four possible pressure levels (Figure 18).

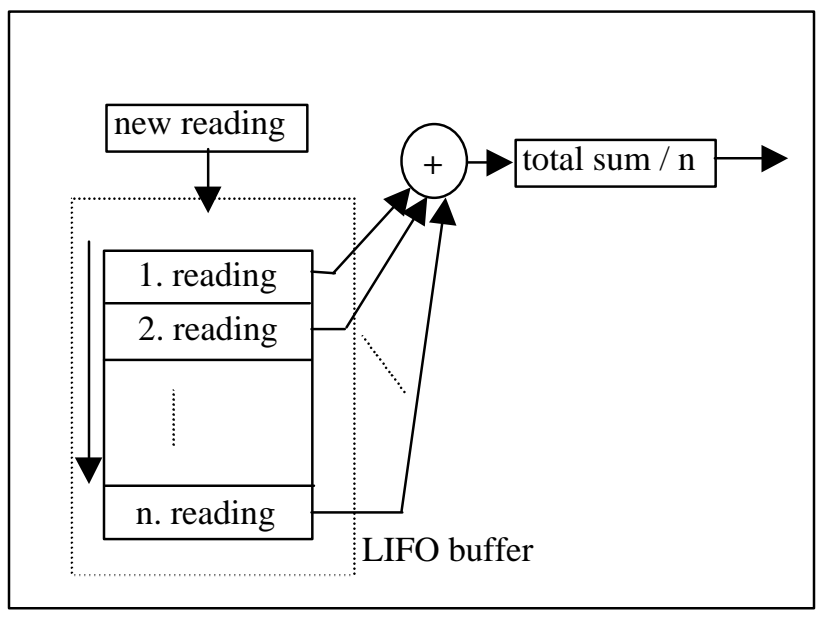

Figure 18. Filtering reading from the sensors

During the examination, the user must cover the entire area of the breast model and have three distinguished levels of pressure at each location. There are three tables used for latching light, medium or deep level of pressure for each sensor. Each place in the table is latched when the user applies and holds a pressure on the same spot for a minimum period of 0.9 seconds. This period is long enough for the user to sense the nodularity of the breast tissue and to identify any breast lump. The latching tables are then used in the evaluation of the user's results. 
Another important issue during the examination is the detection of the user's search pattern. During the palpation, pads of the three fingers are used. Applied pressure on the breast model is registered and displayed on the screen as a wide connected zone. The centroid of the connected zones was used to find a user's search pattern during the examination (Figure 19).

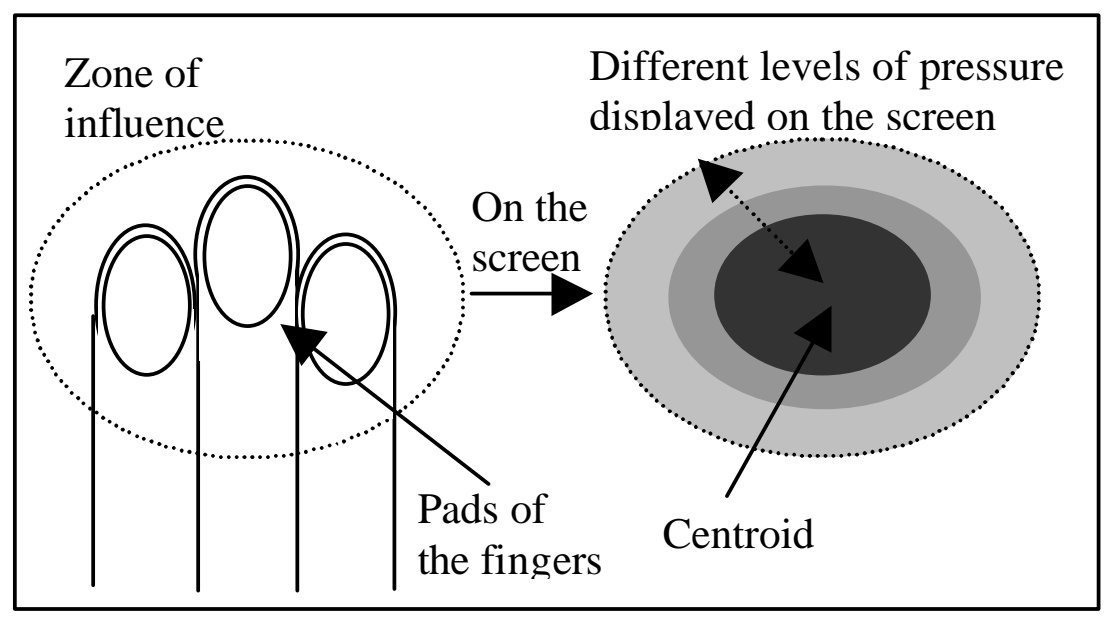

Figure 19 Zone of influence generated from applied palpation

When the palpation is applied on the breast model, a zone of influence is generated. This connected zone is presented visually as a convex area with increasing pressure from the edge to the center of the area. The center of applied palpation or centroid registers the highest level of pressure. This narrow area, in fact, represents the current spot along the search pattern during the examination. Calculating the centroid is the key for locating the exact position of the current spot. Connecting all the registered spots during the examination forms the search pattern. The centroid can be calculated using the equation :

$$
x_{\text {centroid }}=\left(\sum_{n} X i * W i\right) / n \quad y_{\text {centroid }}=\left(\sum_{n} Y i * W i\right) / n
$$


in which $\mathrm{Xi}$ is horizontal position of $\mathrm{i}$-th sensor on the pad, Yi is vertical posiition of the i-th sensor on the pad and $\mathrm{Wi}$ is the value of applied pressure (weight). Once the position of the centroid is calculated, we need to find out the level of applied pressure.

We first need to look for the maximum level of latched pressure within the entire touch pad. After the maximum pressure is being determined, collection of all spots with the same level of applied pressure on the pad is performed. If the user keeps all fingers together (as shown in Figure 19), the spots with the maximum registered pressure are connected, forming one zone. This means that the user is focused on one spot during the palpation. A located centroid is treated as a result of correct palpation and recorded in the table of the user's search pattern. In a case when the user does not keep her / his fingers together, the zone of the maximum applied pressure is disconnected (Figure 20). The calculated centroid can belong to one of those zones or might even be outside all zones. That case is treated as an irregular situation and and the centroid would therefore not be recorded.

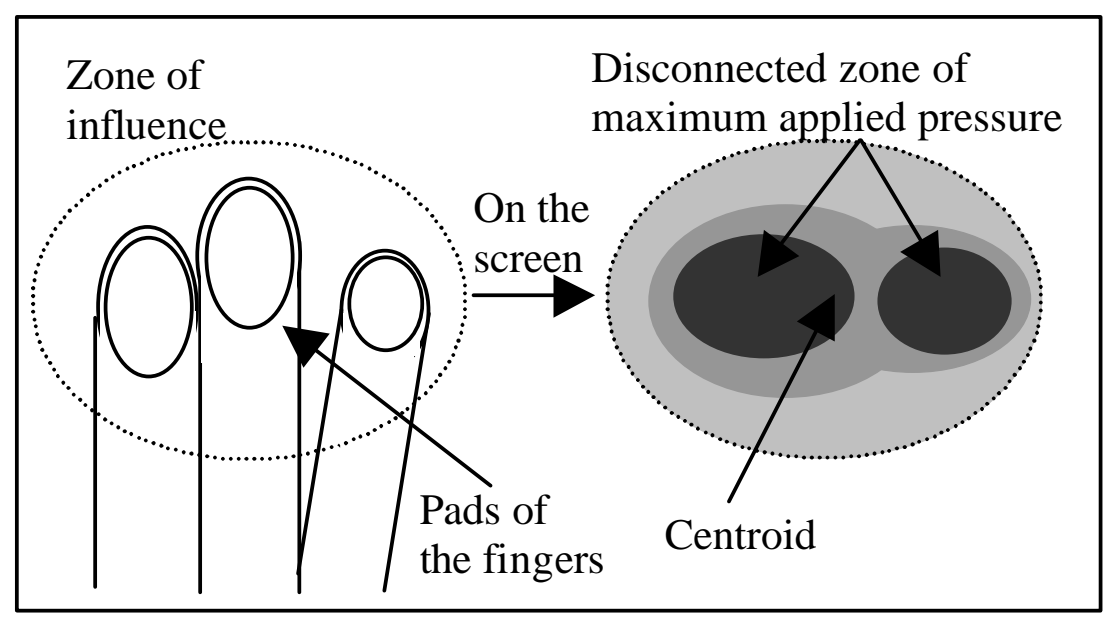

Figure 20 Irregular palpation

Search for the zones of the current maximum applied pressure and their connectivity is processed using 3x3 kernel (Figure 21). All points of current maximum applied pressure are recorded. Algorithm starts with the first recorded maximum and tries 
to find any other recorded point that is connected within a distance defined by kernel (in our case distance is 1) (Figure 22).

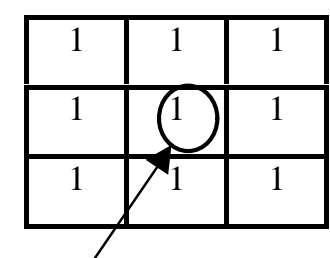

Center of the kernel

Figure 21 Kernel used for search of connected points

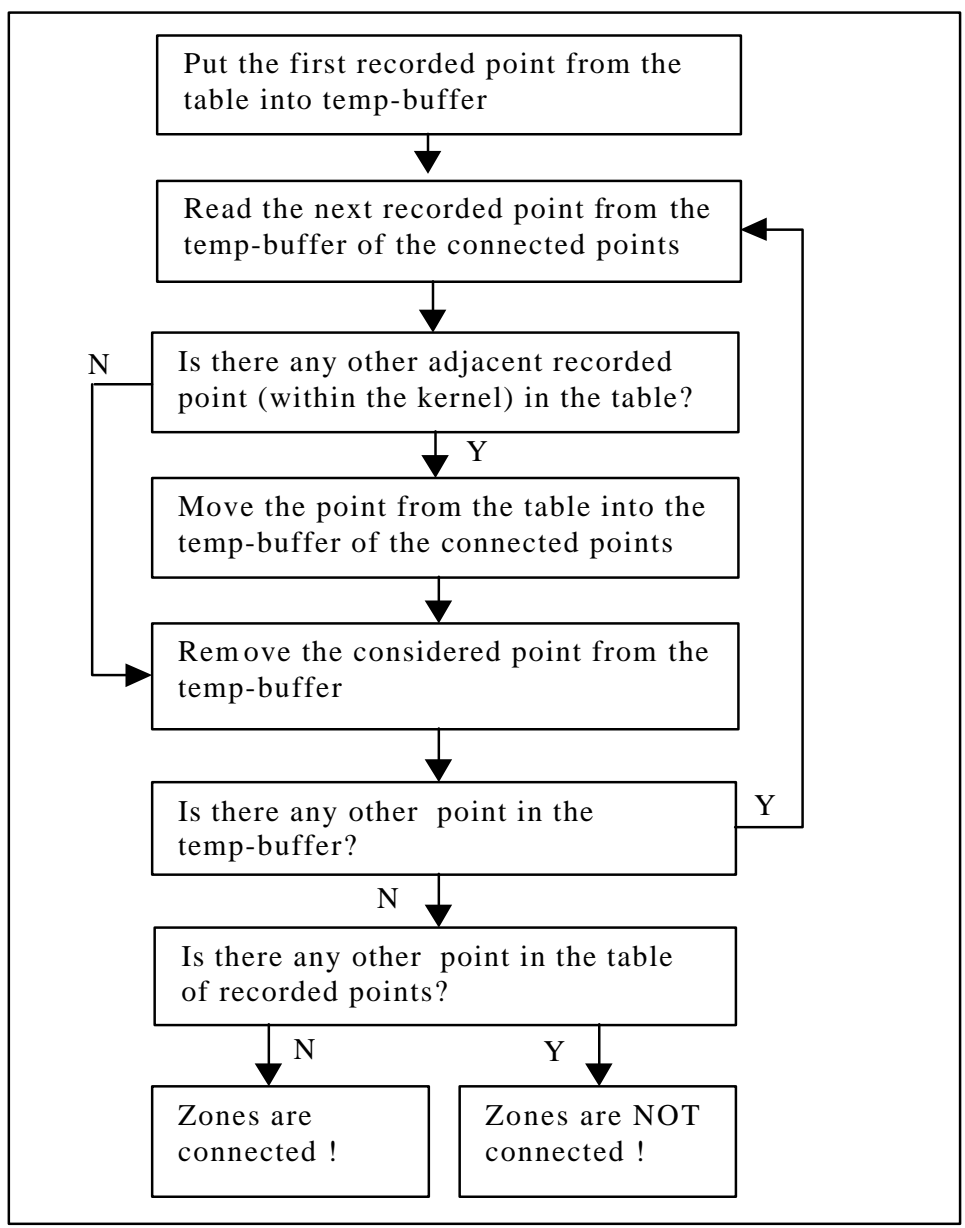

Figure 22 Search algorithm for connected zones 
If found, such point is stored into temporary buffer. The temporary buffer presents a set of currently found adjacent points. In the next step, currently considered point is removed from the temp-buffer and, if the buffer is not empty, next point is considered. Algorithm performs until the last point from the temp-buffer is being processed. If there is any point remained in the table of recorded maximums, it means that there are maximum zones not connected and therefore centroid is not recorded. However, if the table is empty, all zones are connected and the calculated centroid is recorded.

A table of recorded palpations is used for visual presentation of the user's result during the examination. These results are also available in the MS Excel ${ }^{\circledR}$ worksheet table.

It is recommended that the examination last from 120 to 230 seconds for of the entire breast area. This time is long enough for thorough examination and detection of any lump. GUI visual represents the current state of all sensors on the touch pad. The user can terminate the breast model examination at any time by pressing the push button "Done" placed in the GUI.

\subsubsection{Calibration}

The calibration routine is used for setting the properties necessary for correct performance of the entire system. This routine is supposed to be executed in the production process or by technical support. Calibration is related to the touch pad and the breast silicone model properties.

The calibration of the touch pad sensors is available since the visco-elastic properties of the foam change with time. Calibration of the touch pad is performed without using a silicone breast model. The last function is the API function by Tactex Company [13].

The silicone breast model settings include three pressure thresholds and sensor weights. Thresholds of three different levels of pressure (light, medium and deep) can be determined. According to the elastic properties and the three-dimensional oval form of the silicon model, a different table of weights needs to be defined for each new silicone breast model. When the sensor is selected, the user needs to apply the maximum allowed 
light pressure on the selected sensor. When the user is done, a value is recorded. The same procedure issued to calibrate the medium and deep pressure thresholds. Using the three recorded values, a linear function is calculated as well as the sensor's weight factor.

Further development of BETI may contain a database of weight settings for each standard type of silicone breast model that is used.

\subsubsection{Evaluation of results}

The evaluation of results assesses two important factors about the user's performance during the examination of the instrumented model. These are:

1. the effectiveness of three different levels of pressure applied over the entire area of the breast model

2. the similarity between the ideal search pattern and the user's search pattern.

\subsubsection{Level Coverage}

Successful coverage of the instrumented breast area is calculated using the three tables of latched pressures. Each table contains the picture of one level of pressure, that was latched for the sensors of the touch pad. The total number of locations covered for each applied pressure is compared with the ideal - "Experts Performance" table and a relative ratio is derived in percent.

\subsubsection{Sequence}

The user's search pattern is formed when all detected centroids are connected in the sequence of their appearance during the examination. The presentation of the user's search pattern is presented in through the animation and through the plot graph. Compared to the ideal search pattern, the user can locate the areas on the model in which she / he needs to correct or improve her / his performance.

\begin{tabular}{|r|r|r|r|l|}
\hline Hist of palp. & & & & \\
\hline Elap. Time & Row pos. & Col pos. & Norm data & Pressure typ. \\
\hline Seconds & & & & \\
\hline 0 & 1 & 1 & 80 & Light \\
\hline 0.328125 & 1 & 1 & 210 & Light \\
\hline 0.65625 & 1 & 1 & 500 & M edium \\
\hline 0.9375 & 2 & 1 & 310 & Medium \\
\hline 1.20703125 & 3 & 1 & 200 & Light \\
\hline
\end{tabular}

Figure 23 Sample Data File 
This is displayed in the final presentation of the user's results. In addition, recorded data is stored in MS Excel ${ }^{\circledR}$ worksheet that can be used for further analysis (Figure 23).

The rate of success in the applied search pattern is based on the comparison of lengths between the ideal search pattern and the user's one. The touch pad conatins a matrix of sensors arranged in a form 6 rows per 12 columns. Since the vertical strip pattern is used in BSE as the most effective search pattern, 12 columns are grouped into 6 main strips; each two sensor columns wide. Each strip is assigned with a movement direction (Figure 24):

- UP - from the top to the bottom edge of the breast model

- DOWN - from the bottom to the top edge of the breast model

Recorded centroids are used as applied points on the breast model. An algorithm starts from the first recorded centroid and measures the distance form the next one.

According to a assigned direction each sensor within the strip each sensor has different operator used for a distance measurement.

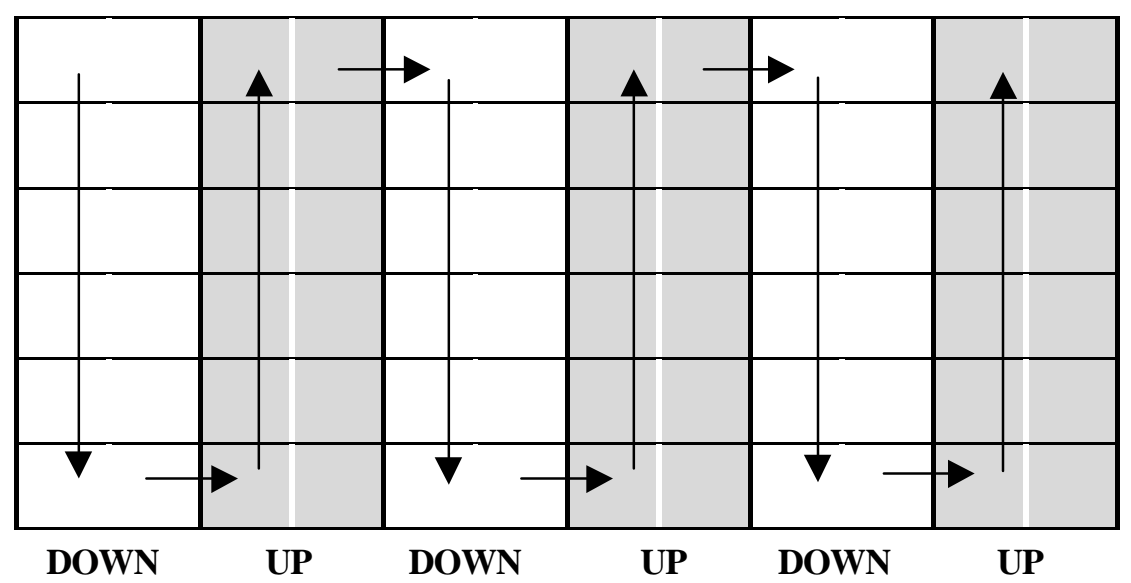

Figure 24 Arrangement of vertical strips and assigned directions 
A distance operator is defined as a $3 \times 3$ matrix described in the Figure $\underline{25}$.

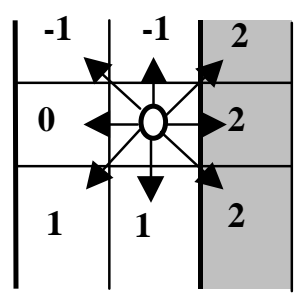

DOWN

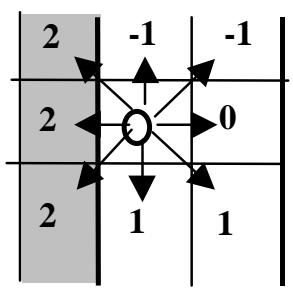

DOWN

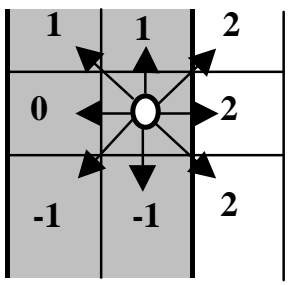

UP

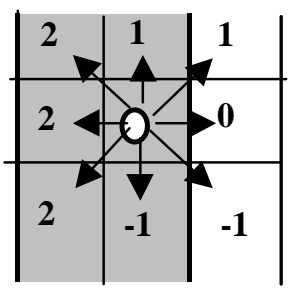

UP

Figure 25 Distance operator according to the movement direction

It can be notice, each the user moves the fingers into the next vertical strip, the distance from the previous centroid increases. Moving within the strip gives the optimized total length of the pattern. When the total length is calculated, it is compared with the length of the ideal search pattern. If the user's search pattern is more close (similar) to the ideal pattern, the difference would be less. Ratio of success in the applied search pattern is based on the calculated search pattern difference and is recorded in the user's results from the examination.

\subsubsection{Presentation of user's results}

A final component of BETI software presents the results of the user's performance during the examination. A set of tabbed pages is presented so that the user would not be confused by a lot of information on one page. The first page shows the user's personal data that was collected from multiple-choice questions in the Personal Risk Estimator (Figure 26). The second page presents the table of user's applied pressures over the entire 
breast area in comparison with the expert's table. The third page shows the user's search pattern through the animation or graph plot.

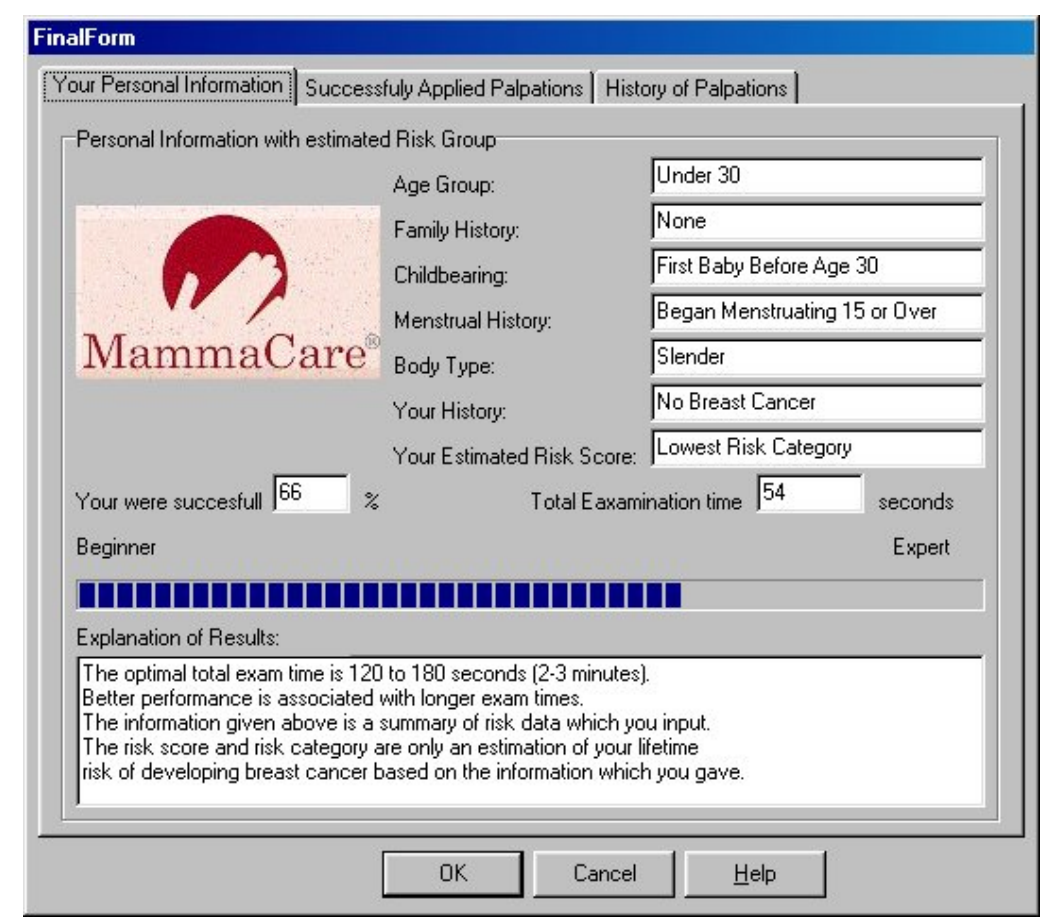

Figure 26 Presentation of the user's results from the examination

\subsection{System Architecture}

The user interacts with the application through the instrumented breast model and GUI.

During the examination and practice, the user applies palpations on the instrumented model. Palpations are recorded as pressure levels applied on the touch sensor device. Data is collected, formatted into Device Data format and sent to the PC. Using Received data is filtered and stored into the table of current sensor values (Figure 27). Device Data can contain an information about the touch device configuration (Figure 29). The touch sensitive device is controlled thorough the Device Command. The Device Command contains a set of commands that supports a full control of the instrumented 
breast model. Figure 28 presents in detail the Device Command format and an instruction set.

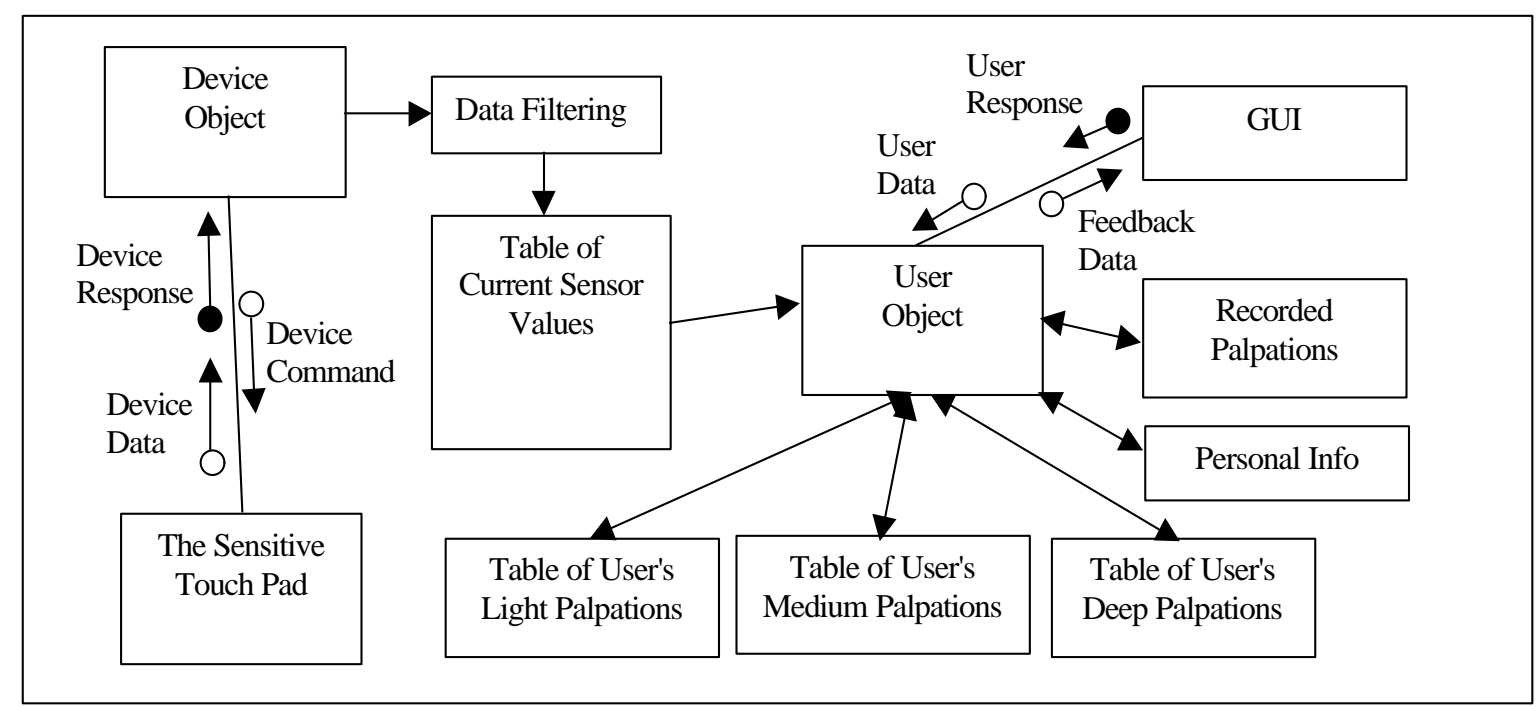

Figure 27 System Architecture

During the examination on the breast model, the user enters personal information, using the GUI. Personal information is sent to the user's object, created with the beginning of the examination, and recorded. After the examination the user's evaluation is completed, GUI is used for the presentation of the user's results (Figure 27). The results are sent to the GUI in the Feedback Data format. Figures $\underline{30}$ and $\underline{31}$ present more details on Feedback Data and User Data.

Device class with its implemented functions presents the touch device and therefore performs communication with the same. Device object acquires data and records them. The other class Client is used to present the current user of the application. Using Device functions, Client reads the current pressure data from the touch pad and records it.

New client object is instantiated when the user starts the breast examination procedure. As an inherited class of the public Device class, instantiated Client object is previously filled with a user's personal information from the Breast Cancer Risk Estimator form. When the user selects real-time pad, communication between client 
object and device is established. The idea of using two classes Device and Client, is based on two main objectives during the examination.

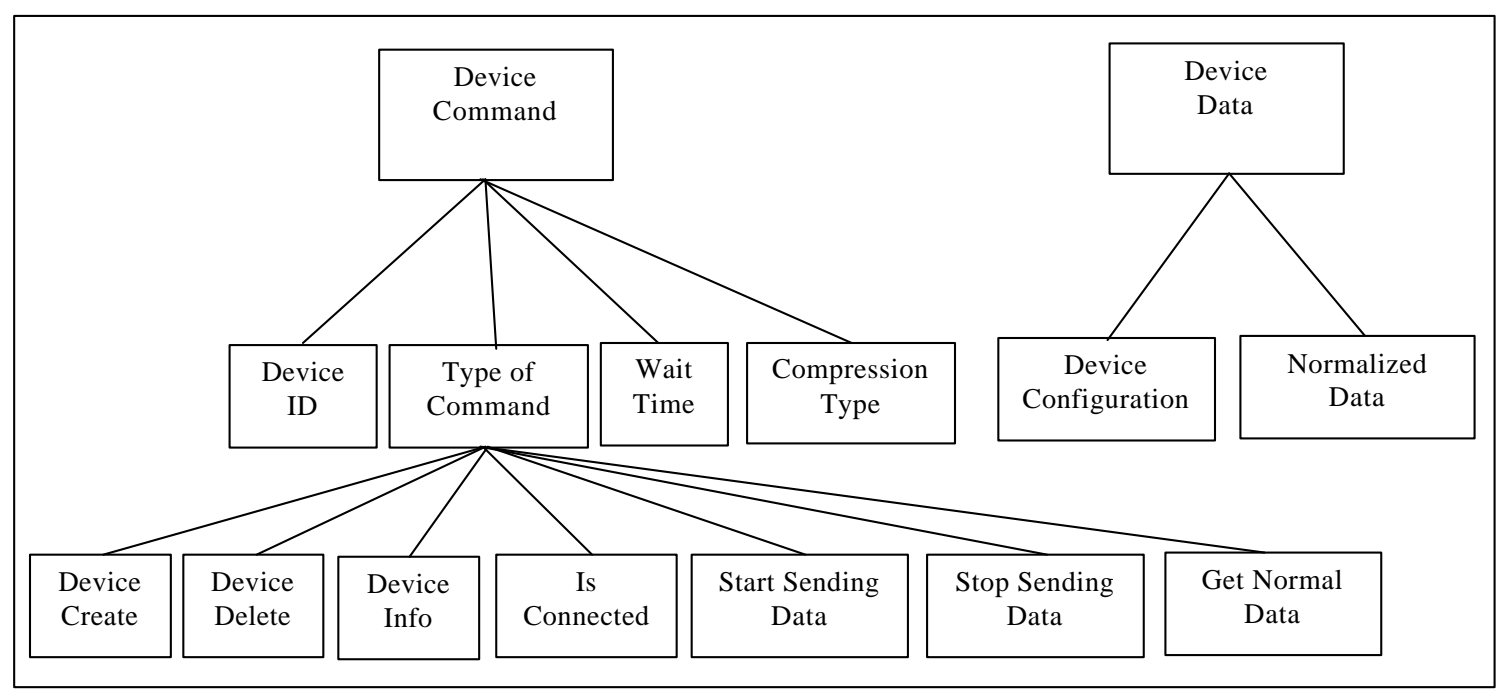

Figure 28 Device Command and Device Data structure

When the examination procedure is done, the user's results are retrieved from the Client object. In addition, user's performance and a risk group are calculated through the implemented Client functions.

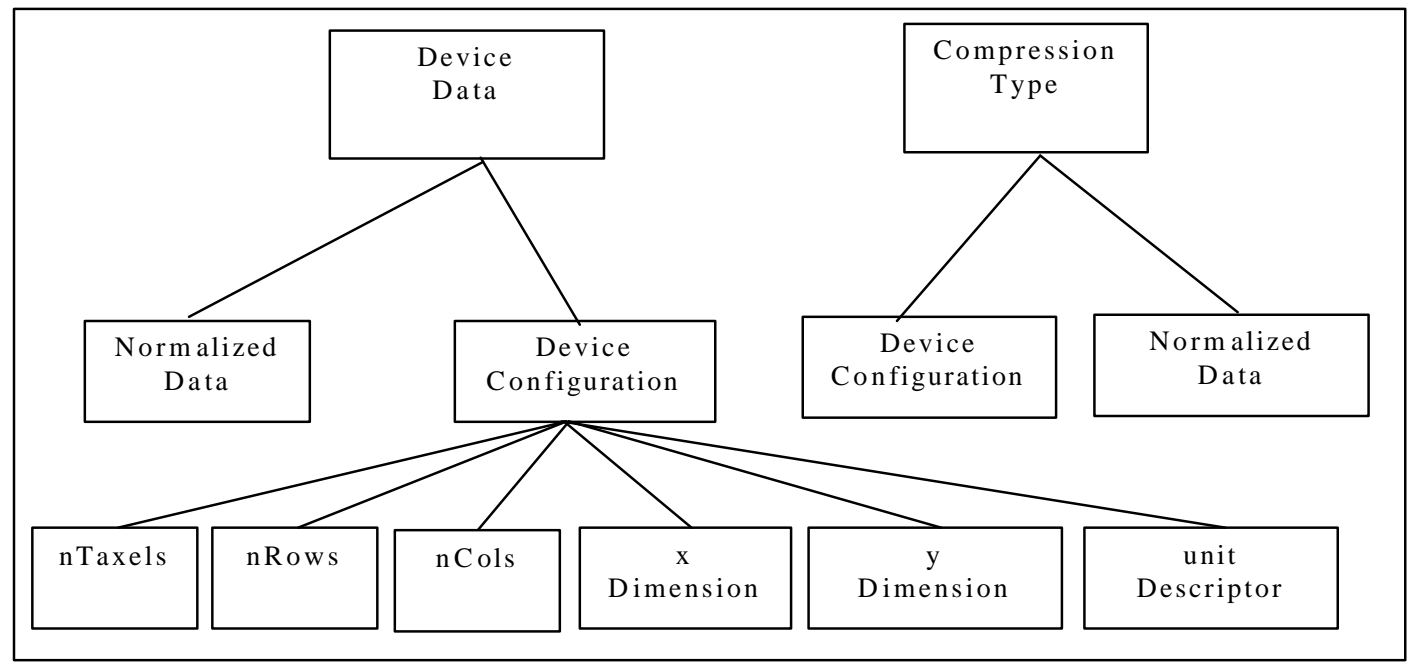

Figure 29 Device Data structure (cont.) 


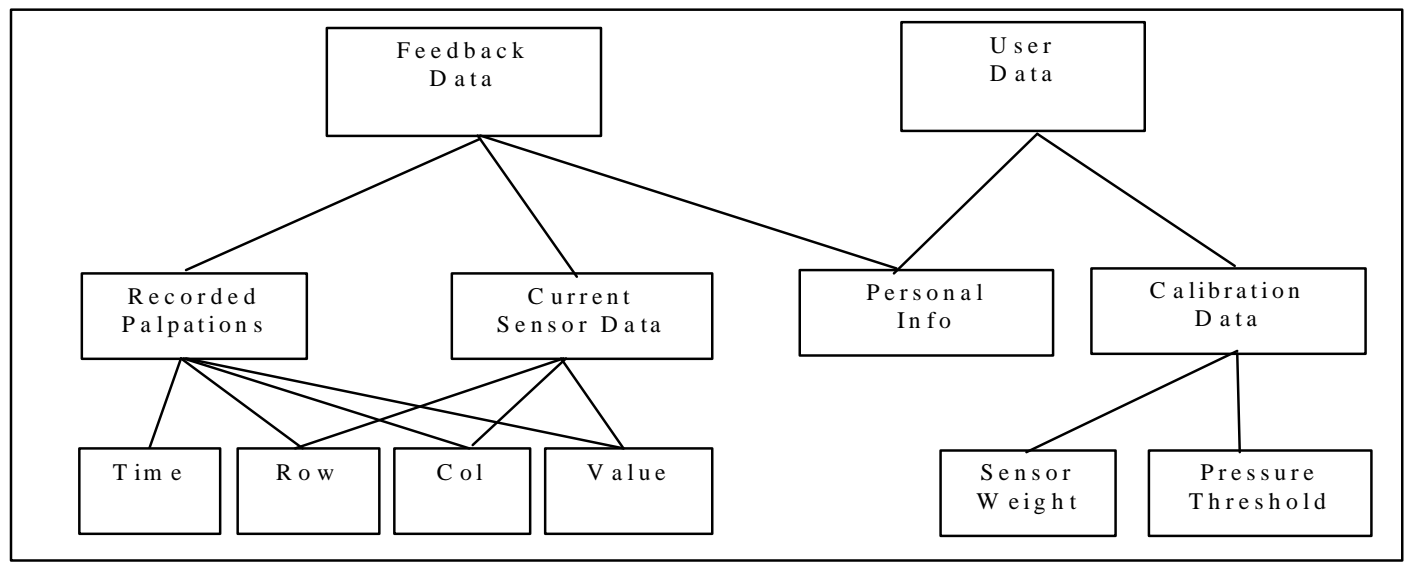

Figure 30 Feedback Data and User Data structure

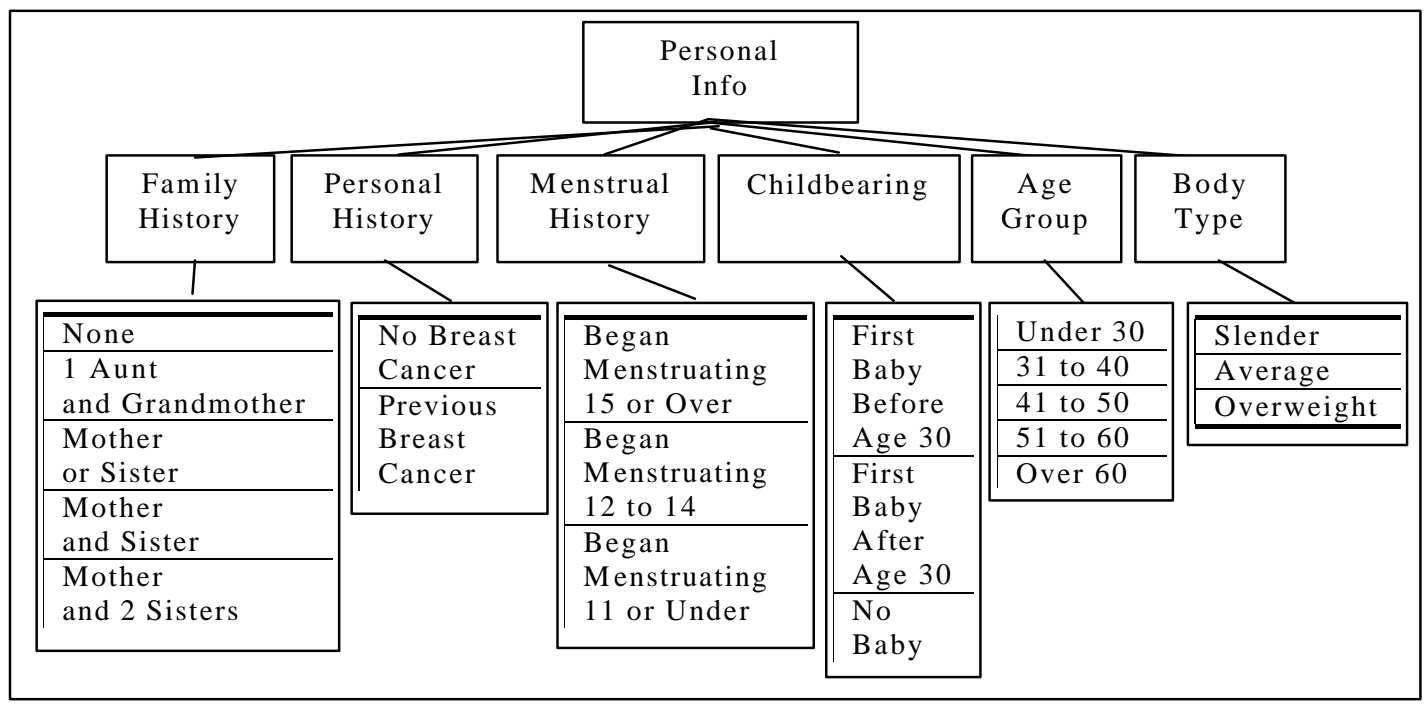

Figure 31 Personal Info structure

\subsection{System operation}

The BETI application is initialized with a procedure that checks that a touch pad device is continued. If the device is not found, the program informs the user about the missing device and terminates. If the device is found, the main menu is opened with four 
main components: an interactive training video, a power point presentation, model breast examination, and a calibration of the instrument (Figure 32).

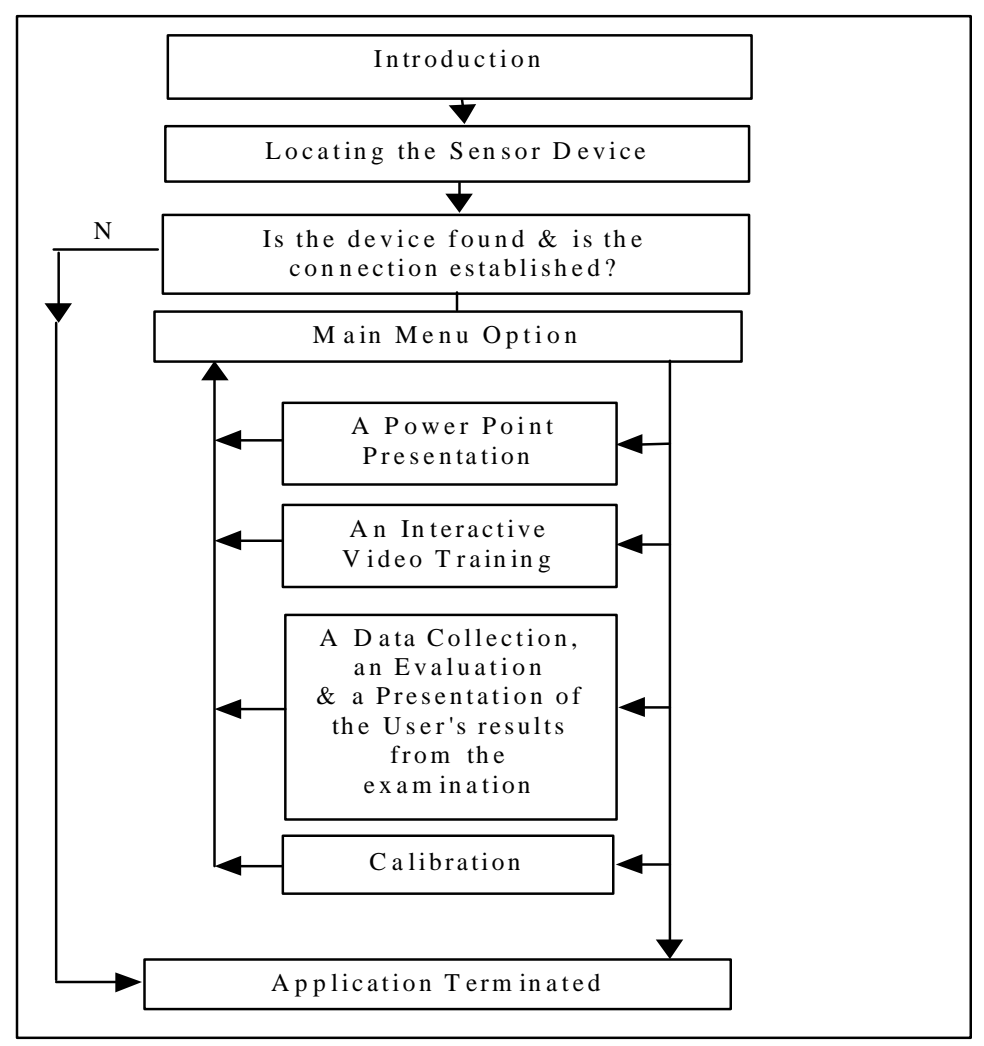

Figure 32 System Operation

Choosing the training video, the user is forwarded to the next video menu that contains a list of topics on the video material. Simply click on any button, and a video fragment will be opened and presented through the Windows Media Player interface. The user may stop video training at any time and go back to the main menu. Figure $\underline{33}$ and $\underline{34}$ present more details on interactive training video.

The power point presentation informs the user about breast cancer, about methods used in breast examination for early detection and how to use BETI application. More details about this presentation have been previously described in this chapter.

The breast examination is executed through two consecutive parts: the Personal Risk Estimator and the Online Data Collection (Figure 35). The Personal Estimator 
places six multiple-choice questions related to the factors that may increase the risk of developing breast cancer. The online data collection is processed during the user's examination of the instrumented model.

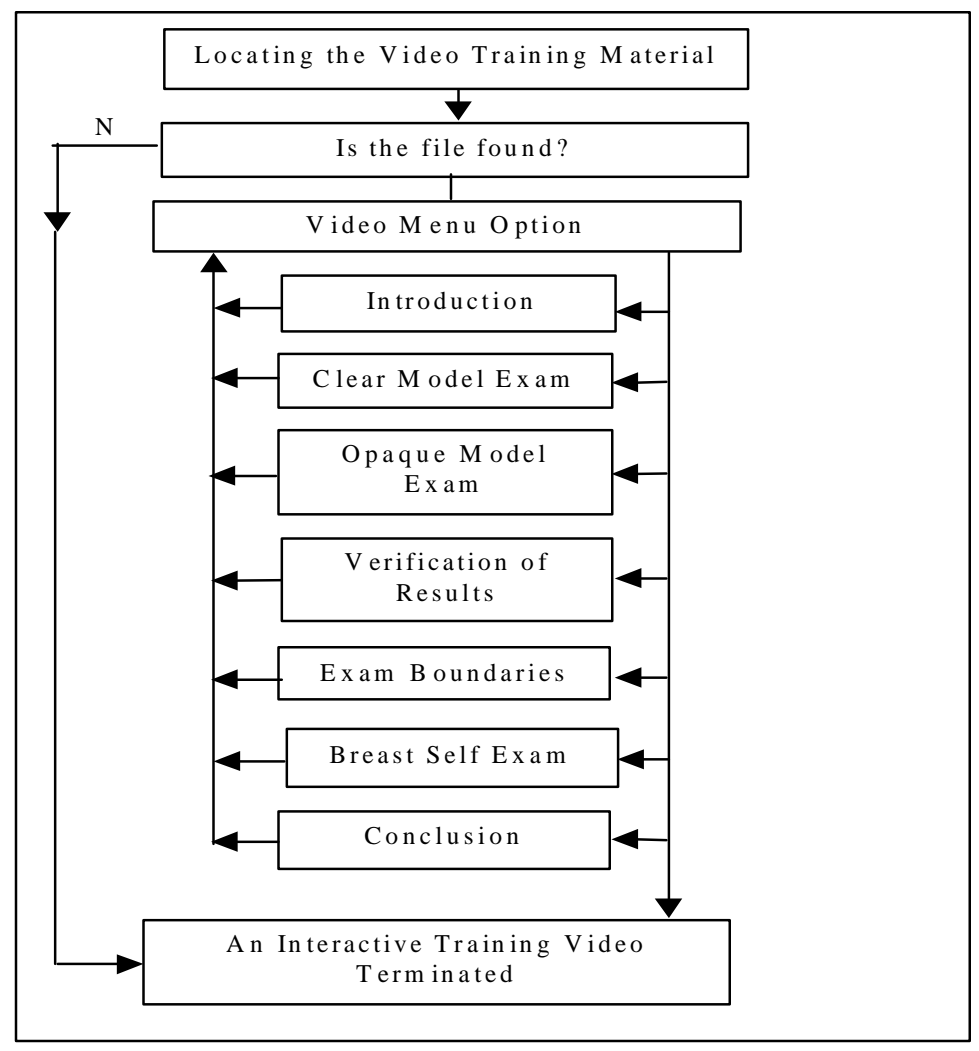

Figure 33 Training Video state diagram

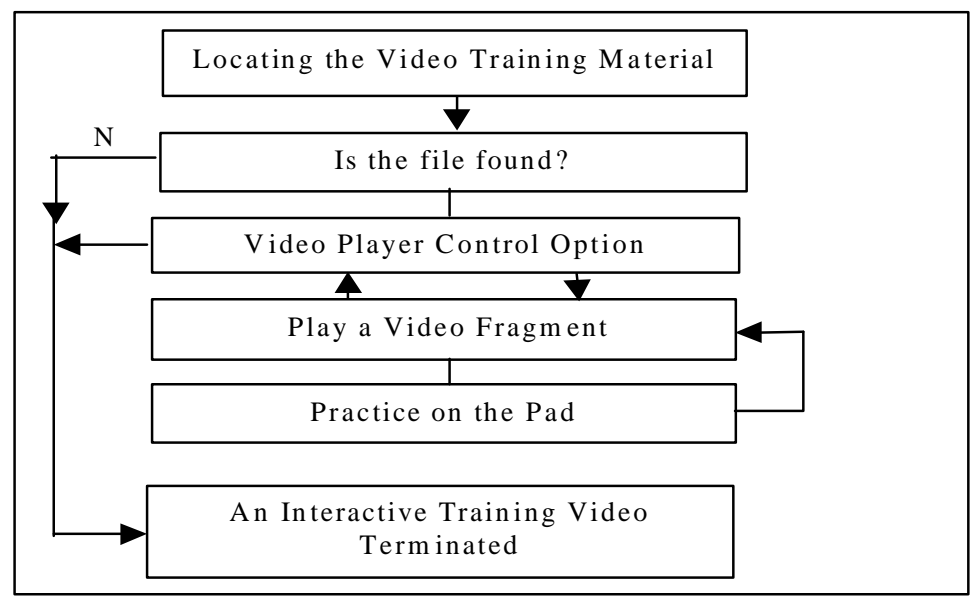

Figure 34 Video Topic Play state diagram 


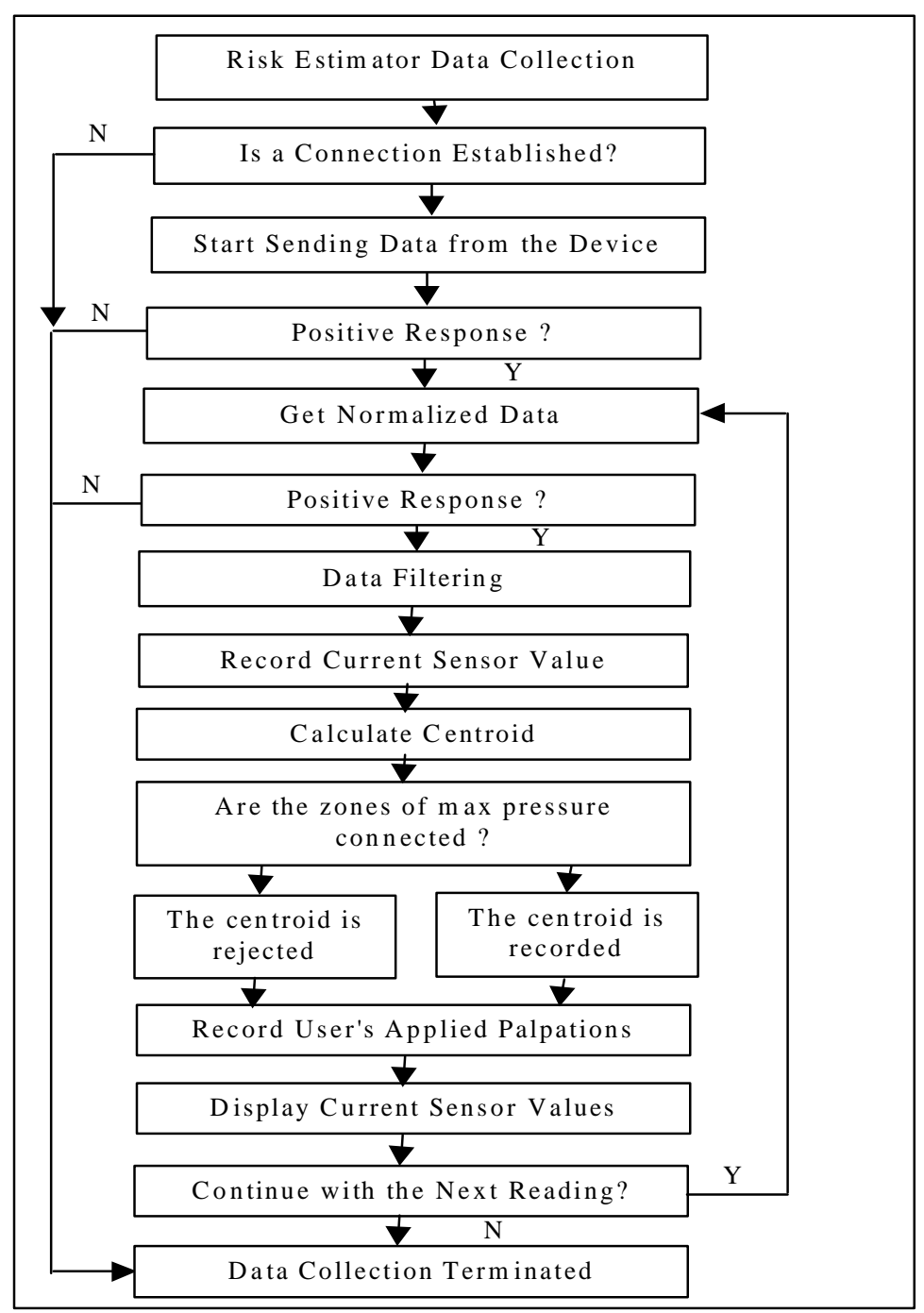

Figure 35 Breast Examination state diagram

The program communicates with the touch pad through the serial connection and reads the actual values from the touch pad sensors. The data is recorded and used for evaluation of the user's performance. The user may cancel the Breast Examination process at any time and go back to the main menu. If the user decides to resume this procedure, the evaluation of the user's results is initiated. This operation is followed by a presentation of her / his success during the examination and an estimation of the user's risk group. MS Excel ${ }^{\circledR}$ worksheet table is generated and opened for further use and analysis. At any time, the user can choose the main menu and terminate the application. 


\section{CHAPTER FIVE}

\section{SYSTEM SUMMARY}

System integration ensures communication between the instrumented device and the software application. The silicone breast model is attached on the top of the touch sensor device. Applied palpation on the breast model, causes pressure to appear on the pad foam covered with a matrix touch sensors. Detected pressure is recorded inside the touch sensitive device. Remote electronics provides the device with RS-232 serial communication. Using the serial COM port in the PC, the software application reads the data from the device. The read data is recorded and stored into the user's record of palpations, displayed the GUI, and evaluated.

Client COM components for Microsoft Power Point Presentation ${ }^{\circledR}$ and Microsoft Excel ${ }^{\circledR}$ have been developed and integrated to the BETI software. These components provide user's tutorial and worksheet analysis of the user's results from the examination.

Communication between the user and BETI application is proceeded through the one of two available ways:

1. the instrumented breast model

2. the user-friendly GUI

The instrumented breast model provides user's input in a form of applied palpations during the training procedure and the breast model examination. The GUI supports user's input with an easy operation of a computer mouse and the output with a status of the application, a status of the touch sensitive device, and the evaluated user's results from the examination.

The developed system represents fully automated training instrument on BSE techniques. An interactive training video, an examination on the instrumented breast model and a non-subjective evaluation of the user's results from the examination are the key components that make the BETI project highly sophisticated educational instrument. 


\section{CHAPTER SIX}

\section{CONCLUSION}

\subsection{New Prototype Status}

As a newly-launched project, BETI is still "a work in progress". Although new instrumented devices now supersede the initial model, developed by Dr. Susan Leight and later improved by Christy Schmidt, many substantial improvements have been made from the previous prototypes. These changes include: a cheaper and more reliable touchsensitive device, software routines that are integrated into a Windows software package, increased user interaction, a more precise algorithm for data processing and evaluation, as well as an improved visual presentation of the user's performance.

A more realistic silicone breast model is used as an attached component to the touch sensitive device. The newly designed model improves the quality of touch perception. The shape of the model, its texture with representative silicone breast tissue, and a set of artificial lumps that are arranged inside the model all contribute towards a more natural look and feel to the examination training.

The touch sensitive instrumented breast allows BETI to communicate with any PC, which has a serial communication port. The matrix of opto-electric sensors, embedded in polymer foam (urethane, silicone, styrene), provides a precise location of the user's finger position and the palpation level on the silicone breast model. The sensors inserted in the touch pad foam are chemically inert, immune to EMI, non-conductive, and non-corrosive. The touch pad foam itself is relatively durable, attachable to any oval three-dimensional shape and available in custom designed size. The device pad and the breast model can be calibrated easily through the automated procedure included in the BETI application. Finally, the cost of the touch pad is one sixth of the cost of the previous prototype sensor system. The new instrumented breast model is smaller and lighter and can be easily transported and setup. 
A Windows software application controls the touch sensitive device without any user intervention except for the installation. The interaction between the user and the software application is performed and presented through a user friendly on screen menu. Analysis and design of software were created using an Object Oriented approach. An interactive training procedure gives the user feedback through visual display of her / his actions. Practice on the instrumented model during the training phase familiarizes the user with the breast model. An evaluation of the user's skills includes an analysis of the user's search pattern during the examination. The search pattern is a key to successful $\mathrm{SBE}$ and is new in this prototype.

\subsection{Conclusion}

The newly developed BETI project has improved the previous prototype.

A new touch sensor has been found and used in an instrumented breast model. The sensor is considerably more flexible and reliable than the previous model. The touch sensor detects and locates the palpation more precisely. In addition, the new sensor material is much lower cost than the sensor used in the previous prototype. The smaller size of the sensor makes the whole training instrument portable.

BSE techniques have been implemented in the interactive training process, the user's examination and the non-subjective evaluation of the user's results. The evaluation of the user's results is more precise. A visual presentation during the interactive training process, the examination, and presentation of the results is more detailed and helpful.

Finally, system integration of the breast model device and software has produced fully automated Windows application, which can be easy operated by anyone who is literate and can use a computer mouse.

\subsection{Future Work}

While the BETI project has made improvements from the original design, there could still be work to be done. Some previously mentioned areas would include designing 
another sensor system. Some of the solutions described in the previous section should be considered for further development of the touch sensor device.

The user's interaction with the application could be improved with controls installed on the very touch device. That would make the user focus on the instrumented model and decrease confusion that may come from the available controls on the screen.

The training process should include detection of the artificial implanted lumps. This procedure increases both the user's learning rate and potential in successful examination. During the examination, the user would use separate control (pushbutton installed on the touch pad) and press it whenever she/he feels it. Those points should be recorded and matched with actual positions of the artificial lumps. A measured distance would indicate successful palpation during the examination.

The use of OpenGL would provide a three-dimensional graphic presentation of the instrumented breast model on the screen. The user would certainly envision a better presentation this way during the training and examination procedure.

The touch sensor device should also allow the use of several breast models. For each model, properties such as size, thickness, arrangement of the lumps within the model would be stored in local database. The use of a database would decrease the need for new calibration whenever the breast model is changed. Using a variety of different breast models would allow the user to gain mastery in a variety of conditions. 


\section{BIBLIOGRAPHY}

1. Leight, Susan. "The Use of an Instrumented Breast Model for the Examination of Patterns of Search in Breast Self-Examination.” Unpublished Doctoral Dissertation. West Virginia University, Morgantown, WV. (May, 1995).

2. Landis, Sarah H., Taylor Murray, Sherry Bolden, Phyllis A. Wingo. "Cancer Statistics, 1998." CA - A Cancer Journal for Clinicians. 48.1 (January/February 1998): 6-29.

3. University of Wisconsin Comprehensive Cancer Center [UWCCC]. "Breast Cancer Awareness: Frequently Asked Questions, Screening." (December 1998). Online. World Wide Web. http://www.medsch.wisc.edu/bca/faq/screening.html. 14 February, 1999.

4. Hall, Deborah H., Calvin K. Adams, Gerald H. Stein, Hester S. Stephenson, Mark K. Goldstein and H. S. Pennypacker. "Improved Detection of Human Breast Lesions Following Experimental Training." Cancer. 46 (15 July, 1980): 408-414.

5. Newcomb, P.A., Wiess, N.S., Storer, B.E., Scholes, D., Young, B. E., \& Voight, L.F. (1991). Breast self-examination in relation to the occurence of advanced breast cancer. Journal of the Natioanal Cancer Institute, 83, 260-265

6. Fletcher, Suzanne W., Michael S. O'Malley, Leslie A. Bunce. "Physicians' Abilities to Detect Lumps in Silicone Breast Models.” JAMA. 253.15 (19 April, 1985): 2224-2228.

7. Anthony B. Miller, Teresa To, Cornelia J. Baines, Claus Wall "Canadian National Breast Screening Study-2: 13-Year Results of a Randomized Trial in Women Aged 50-59 Years". Journal of National Cancer Institute, Vol. 92, No 18, (20 September, 2000): 1490-1499

8. Pinto, B., \& Fatiqua, R.W. (1991). Training breast self-examination: A research review and crititque. Health Education Quarterly, 18(4), 495-516

9. Saunders, Kathryn J., Carol A. Pilgrim and Henry S. Pennypacker. "Increased Proficiency of Search in Breast Self-Examination." Cancer. 58 (1 December, 1986): 2531-2537. 
10. Christy Schmidt, M.S.E.E., "The Development of the Breast Examination Training Instrument", Master Thesis, West Virginia University (1999).

11. E.M. Reimer, President, Canpolar East Inc., L.H. Baldwin, Product Manager, Canpolar East Inc. "Cavity Sensor Technology for Low Cost Automotive Safety \& Control Devices" (14 February 2000).

12. Mammacare Company, "Method of Breast Self-Examination", VHS video matrial (2000).

13. Tactex Controls Inc, "MTC Express - Owner's and Developer's Guide", version 1.1 (20 April 2000).

14. Borland Corporation, "Borland C++ Builder v.5.0 User Manual"

15. Health EdCo tri-fold display entitled "Caring for your breasts"

16. Greenlee, Murray, Bolden, \& Wingo, 2000 “Cancer Statistics, 2000.” CA - A Cancer Journal for Clinicians.

17. Kim, Taehee, Malcolm, C. A, Hallam, John "Developing of vibration sensors as event signature sensors in assembly", University of Edinbourgh, 1996

18. Powers, William B, "Piezo film sensing devices", Society of Manufacturing Engineers", 1986

19. Broadhurst, Martin G, "PVDF and associated piezoelectric polymers", Gordon and Breach Science Publishers, 1981

20. Chiu, Yih-Nong, "Tactile sensor and strain gauge in a robotic gripper system", Huntsville, AI., 1985

21. Dimitris Hristu, Nicola Ferrier, Roger W Brockett, "The performance of the deformable-membrane tactile sensor: basic results on geometrically -defined tasks", IEEE, Conf. on Roboticsand Automation, 2000

22. Interlink Electronics Corp., "Integration guide on VersaPad and VersaPoint", Publication \#90-65801-Rev. B, 1998

23. MSI Corp. "Applications of Piezo Cable", Sensors Product Division (Europe) RB 10/99 